\title{
From vacuum fluctuations across an event horizon to long distance correlations
}

\author{
Renaud Parentani* \\ Laboratoire de Physique Théorique, CNRS UMR 8627, \\ Bât. 210, Université Paris-Sud 11, 91405 Orsay Cedex, France
}

(Dated: June 4, 2018)

\begin{abstract}
We study the stress energy two-point function to show how short distance correlations across the horizon transform into correlations among asymptotic states, for the Unruh effect, and for black hole radiation. In the first case the transition is caused by the coupling to accelerated systems. In the second, the transition is more elusive and due to the change of the geometry from the near horizon region to the asymptotic one. The gradual transition is appropriately described by using affine coordinates. We relate this to the covariant regularization used to evaluate the mean value of the stress energy. We apply these considerations to analogue black holes, i.e. dispersive theories. On one hand, the preferred rest frame gives further insight about the transition, and on the other hand, the dispersion tames the singular behavior found on the horizon in relativistic theories.
\end{abstract}

PACS numbers: 03.75.Kk, 04.62.+v, 04.70.Dy

\section{INTRODUCTION}

In Minkowski space the vacuum of quantum fields, the ground state of the Hamiltonian, is globally defined. For instance, for a massless free field $\phi$ in two dimensions, it is the state annihilated by the destruction operators $a_{k}$ associated with the positive norm modes $\phi_{k}=e^{-i \Omega t+k z} /(4 \pi \Omega)^{1 / 2}$ where $\Omega=|k|>0$. When probed locally, it determines correlation functions, such as that of the stress-energy tensor. Considering $T_{U U}=\left(\partial_{U} \phi\right)^{2}$, where $U(V)$ is the retarded (advanced) null coordinate $U=t-z,(V=t+z)$, the connected function is

$$
\begin{aligned}
\left\langle T_{U U}(U) T_{U U}\left(U_{0}\right)\right\rangle & =\left(\partial_{U} \partial_{U_{0}}\left\langle\phi(U) \phi\left(U_{0}\right)\right\rangle\right)^{2} \\
& =\left(\frac{1}{4 \pi} \frac{1}{\left(U-U_{0}-i \epsilon\right)^{2}}\right)^{2} .
\end{aligned}
$$

The regulator $\epsilon \rightarrow 0^{+}$specifies the nature of the singularity in the limit $U \rightarrow U_{0}$. It arises from the fact that only positive frequency $\Omega=i \partial_{U}$ contribute to Eq. (11).

Even though this function monotonically behaves in $\Delta U^{-4}$, it determines subtle effects. This can be seen by considering accelerated systems where both local and non-local phenomena related to the Unruh effect [1-7] can be obtained from Eq. (11). Moreover the correlations of $T_{U U}$ in the (Unruh) vacuum across the future horizon of a black hole behave as in Eq. (1). However since the black hole geometry is non uniform, as one probes the vacuum further away from the horizon, there is a gradual change which results, on one hand, in correlations typical of a thermal flux at the Hawking temperature [3, [8, 9] and, on the other, in correlations across the horizon between Hawking quanta and their partners [10].

The aim of the present paper is to analyze the black hole case in the light of the Minkowski case. We show that the gradual change in the correlations comes through the observables used to probe the state, and not from $\phi$ which is conformally invariant and thus insensitive to the geometry. We relate this gradual change to that obtained when considering renormalized expectation values which also depend on the conformal factor. In the last part, we consider dispersive field theories [11], or analogue black holes in condensed matter [12, 13]. First, we show that the preferred rest frame [14] associated with dispersion defines new scalars (observables) which give an unambiguous meaning to the gradual change of the stress energy correlations. Second we study how dispersion affects the correlation pattern. ${ }^{1}$ In addition to the robustness of the spectral properties of Hawking radiation [11, 16, 17], that of the long distance correlations across the horizon was established by constructing wave packets of $i n$ modes [16]. We shall return to this observation and make contact with the analysis of density-density correlations in Bose-Einstein condensates [18 20].

*Electronic address: renaud.parentani@th.u-psud.fr

1 While completing this work, we became aware of [15] where similar conclusions are reached. 
We have organized the paper as follows. In Section II, we describe the behavior of the stress energy 2pt function in flat space, and interpret its properties by introducing external (accelerated) systems which probe the field. In Section III, we perform the same analysis in a black hole geometry. Using affine coordinates, we present an invariant description of the transition from its near horizon behavior, which is similar to Eq. (1), to its long distance behavior. Finally, in Section IV, we consider the stress energy correlations in dispersive theories, and investigate what are the modifications induced by dispersion. We conclude with remarks about the similarities between these dispersive effects and those obtained with relativitic fields propagating in fluctuating metrics [21, 22].

\section{UNRUH EFFECT AND CORRELATIONS ACROSS A RINDLER HORIZON}

We introduce the null coordinates $a u=-\ln (-a U), a v=\ln (a V)$, defined respectively for $U<0$ and $V>0$. These are related by $\tau=(u+v) / 2$ to $\tau$, the proper time of an accelerated system which follows $z^{2}-t^{2}=1 / a^{2}$ in $R$, the right quadrant $z>|t|$. ( $a$ is the constant acceleration.) When $U$ and $U_{0}$ are negative, i.e. with both points on the right of $U=0$, the future horizon of the accelerated system, Eq. (1) can be re-written using $u$. Introducing $T_{u u}^{R}=\left(\partial_{u} \phi\right)^{2}=T_{U U} e^{-2 a u}$, Eq. (1) becomes

$$
\left\langle T_{u u}^{R}(u) T_{u u}^{R}\left(u_{0}\right)\right\rangle=\left(\frac{1}{16 \pi} \frac{a^{2}}{\left(\sinh \frac{a}{2}\left(u-u_{0}-i \epsilon\right)\right)^{2}}\right)^{2} .
$$

Since $1 /(\sinh y)^{2}=\Sigma_{n}(y+i n \pi)^{-2}$, where the sum over $n$ goes from $-\infty$ to $\infty,\left\langle T_{u u}^{R} T_{u u}^{R}\right\rangle$ is the periodic function in $\operatorname{Im} u$ of period $2 \pi / a$ whose zeroth term, $n=0$, behaves as in the vacuum, see Eq. (11). Hence Eq. (2) is the 2-point function in a thermal bath at a temperature equal to $a / 2 \pi$. Noticing that along the accelerated trajectory, one has $\tau=u$ (because $v=u$ ) Eq. (2) determines the correlation function of the energy density probed by the accelerated system. In this way we recover the Unruh effect: accelerated systems perceive the vacuum as a thermal ensemble at that temperature [1].

It is less usual but equally interesting to consider Eq. (1) with one point on either side of the horizon. Using $u$ and $T_{u u}$ on the right, and their symmetrical counterpart on the left, $a \bar{u}=\ln (a U)$ and $T_{u u}^{L}=\left(\partial_{\bar{u}} \phi\right)^{2}=T_{U U} e^{2 a \bar{u}}$ for $U>0$, Eq. (1) becomes

$$
\left\langle T_{u u}^{L}(\bar{u}) T_{u u}^{R}\left(u_{0}\right)\right\rangle=\left(\frac{1}{16 \pi} \frac{a^{2}}{\left(\cosh \frac{a}{2}\left(\bar{u}+u_{0}\right)\right)^{2}}\right)^{2} .
$$

Quite surprizingly $\left\langle T_{u u}^{L} T_{u u}^{R}\right\rangle$ possesses a 'bump' which is centered at $\bar{u}+u_{0}=0$. In terms of the affine coordinate $U$, it is located at $U=-U_{0}$, on the opposite value from the horizon. This is unnexpected because, at fixed $\Delta V$, $\left\langle\phi(U, V) \phi\left(U_{0}, V_{0}\right)\right\rangle=\frac{-1}{4 \pi} \ln \Delta U+C s t$. has no maximum when expressed in terms of $\bar{u}$ and $u_{0}$. In fact, the maximum in Eq. (3) results from the combined effect of the Jacobians $d U / d u$ associated with the tensorial character of $T_{U U}$, and the monotonic power law decay of Eq. (11). ${ }^{2}$ These properties apply to massless fields in any space-time dimension $d$. Because of the dimensionality of the $\phi$ field $(=(d-2) / 2)$ the location of the maximum is shifted at $\bar{u}+u_{0}=$ $-\frac{2}{a} \operatorname{arctanh} \frac{d-2}{d+2}$.

So far however Eq. (3) is simply a re-expression of Eq. (1): no physics is gained when writing a tensor in a new coordinate system. So, isn't the maximum of Eq. (3) just a coordinate artefact? No, Eq. (3), as Eq. (2), governs physical effects. For instance, when looking at the state of two opposite accelerated systems 7], the correlation matrix of this bi-partite system exhibits a non-trivial entanglement near the maximum of Eq. (33). Similarly the scattering by two opposite accelerated mirrors destructively interferes near that maximum [6]. As another example [4], expressions closely related to Eq. (3) obtain when looking at the value of $T_{u u}^{L}$ which is correlated to the fact that an accelerated detector in $R$ has made a transition. In all cases the physics is the same: measurements performed in $R$ are statistically correlated to those performed on the other side of the horizon.

To convey the idea that the re-writing of Eq. (11) as Eqs. (2, 3) is not merely a coordinate change but has to do with the physics of accelerated systems, we make two observations. First it is conceptually useful to introduce the ( $U$ part of the) scalar energy density that such system in $R$ will measure: $\rho^{R}=T_{\mu \nu} u_{R}^{\mu} u_{R}^{\mu}$, where $u_{R}^{\mu}=d x_{R}^{\mu} / d \tau$ is the unit vector field tangeant to its trajectory. Then the l.h.s of Eq. (2) is $\left\langle\rho^{R}(\tau) \rho^{R}\left(\tau_{0}\right)\right\rangle$. Similarly the l.h.s of Eq. (3) is $\left\langle\rho^{L}(\bar{\tau}) \rho^{R}\left(\tau_{0}\right)\right\rangle$. Hence both Eq. (2) and Eq. (3) have a clear interpretation as energy-energy correlation functions of

\footnotetext{
2 My attention was drawn to this point in a discussion with W. Unruh and T. Jacobson that took place during the workshop "Towards the observation of Hawking radiation in condensed matter systems" held at IFIC in Valencia in Feb. 2009.
} 
accelerated systems. Secondly, we notice that Eq. (2) only depends on $u-u_{0}$. This results from the invariance of the vacuum under Lorentz transformations $u \rightarrow u+b, U \rightarrow U e^{-a b}$. In fact the Minkowski distance between two points situated along the accelerated trajectory $v=u$ is

$$
s^{2}=-\Delta t^{2}+\Delta z^{2}=-\frac{4 \sinh ^{2}\left(a\left(\tau-\tau_{0}\right) / 2\right)}{a^{2}} .
$$

We therefore see that the stationarity of Eq. (2) in $u$ for arbitrary values of $v$ are related to the properties of $s^{2}(\Delta \tau)$ evaluated along $v=u$. In a similar fashion, Eq. (3) is related to the distance $s_{\text {op }}^{2}$ bewteen two points situated along the opposite accelerated trajectories defined by $z^{2}-t^{2}=1 / a^{2}$. Using the proper time $\tau_{0}(\bar{\tau})$ to localize the point on the right (left) trajectory, one gets

$$
s_{\mathrm{op}}^{2}=\frac{4 \cosh ^{2}\left(a\left(\bar{\tau}+\tau_{0}\right) / 2\right)}{a^{2}} .
$$

The maximum of correlation in Eq. (3) thus coincides with the minimum (space-like) distance bewteen the two trajectories. Because of Lorentz invariance no preferred spatial distance $\Delta z^{2}$ is selected though.

Yet, in preparation for the black hole problem, one would like to know what is the origin of the mathematical properties of Eq. (2) and Eq. (3). In fact, these must be deeply rooted since Eq. (3) is the analytical continuation of Eq. (2) obtained by subtracting half of the imaginary period that fixes the Unruh temperature. In addition, we wish to identify what could explain the maximum of correlation in Eq. (3), which is independent of $\Delta V$, and thus not necessarily refers to Eq. (5) and accelerated systems in $L$. As shown in the Appendices, these properties can be understood when using the Unruh modes [1]. In brief, the maximum of Eq. (3) finds its origin in the entanglement in Fock space between states with opposite Killing frequency $\omega=i \partial_{u}$. This entanglement translates into space-time correlations because, in each pair, the partner mode lives on the other side of the Rindler horizon, and is weighted in Eq. (A2) by a factor $z_{\omega}$ which is real for all $\omega$. In addition the maximum value of correlations is directly related to the mean occupation number of Rindler quanta.

\section{HAWKING EFFECT AND CORRELATIONS ACROSS THE HORIZON}

We have a double aim. First, we wish to interpret the equivalent of Eq. (1) and Eqs. (2)3) in a black hole geometry. Second, we aim to analyze the differences between the black hole case and Minkowski. In particular we are looking for a smooth and coordinate invariant interpolation from the near horizon region, where Eq. (1) should make sense, to the asymptotic regions where Eqs. (23) should do.

To reach these ends, we need to identify what corresponds to the Minkowski vacuum, the affine coordinate $U$, and the $u$ coordinate associated with boosts $u \rightarrow u+b, U \rightarrow U e^{a b}$. For simplicity we shall work with a two dimensional conformally invariant field $\phi$. Then the identification is rather easy and known [1, 5]. The novelty mainly consists in the attention paid to the stress-energy correlation function.

\section{A. Prelude: conformal invariance}

We work with a conformally invariant field because the simplicity of the expressions will oblige us to identify under which conditions identities valid in all space-times acquire a physical meaning related to the Unruh and/or to the Hawking effect.

In two dimensions the line element can always be written in double null coordinates as

$$
d s^{2}=-C(U, V) d U d V
$$

In these coordinates, $\phi$ obeys $\partial_{U} \partial_{V} \phi=0$. Therefore the conformal factor $C$ drops out and the right and left moving sectors remain decoupled $\phi=\phi(U)+\phi(V)$. As a result, for any $U$ coordinate, one can consider the $U$-vacuum state defined by the positive frequency modes $\phi_{\Omega}=e^{-i \Omega U} /(4 \pi \Omega)^{1 / 2}$. In that state, the connected correlation function of $T_{U U}=\left(\partial_{U} \phi\right)^{2}$

$$
\left\langle T_{U U}(U) T_{U U}\left(U_{0}\right)\right\rangle_{c} \equiv\left\langle T_{U U}(U) T_{U U}\left(U_{0}\right)\right\rangle-\left\langle T_{U U}(U)\right\rangle\left\langle T_{U U}\left(U_{0}\right)\right\rangle
$$


obeys Eq. (1), independently of the regularization scheme used to compute $\left\langle T_{U U}(U)\right\rangle .^{3}$ Introducing the coordinates $a u=\ln (-a U)$ and $a \bar{u}=\ln (a U)$ for respectively negative and positive values of $U$, Eq. (2) and Eq. (3) also follow, as mathematical identities valid in all space-times and for any coordinate $U$. Some physical input is therefore needed to transform these identities into meaningful relations among observables.

To relate Eq. (2) to the Unruh effect is straightforward. It suffices to consider a particle detector following the orbit $u=v$. It will perceive the $U$-vacuum as a thermal state at a temperature $a / 2 \pi$. (In general $a$ is no longer the proper acceleration, consider e.g. de Sitter space where the thermal bath is also perceived by inertial detectors.) Similarly, for Eq. (3), when introducing another detector which follows the mirror trajectory $\bar{u}=\bar{v}$, the combined state of these two detectors will be entangled as that of accelerated detectors in Minkowski 7], again as a direct consequence of the conformal invariance of $\phi$. This generalization of the Unruh effect applies to any field and in any dimension, but only approximatively provided the acceleration is much higher that the space-time curvature. (This limit is also used in the thermodynamic analysis of space-time [23]. However the fact that Eq. (3) always applies indicates that the purity of the quantum state, and not only the mean fluxes, is a key ingredient.)

To relate Eq. (21) and Eq. (3) to the Hawking effect is more elaborate. As we shall see, the metric plays a crucial role in "selecting" the $U$-vacuum and the coordinate $u$. This follows from the fact that, unlike the connected $2 \mathrm{pt}$ function of Eq. (7), other observables are not conformally invariant, as for instance the renormalized expectation value of $T_{U U}[9]$

$$
\left\langle T_{U U}\right\rangle^{\mathrm{ren}}=-\frac{1}{12 \pi} C^{1 / 2} \partial_{U}^{2} \frac{1}{C^{1 / 2}} .
$$

In Sec. III.D, we shall see that the breakdown of the conformal invariance comes from the renormalization scheme and not from the "bare" operator.

\section{B. The correspondence}

We work with one dimensional stationary black hole metrics that we describe using Eddington-Finkelstein (EF) coordinates $v, r$ in which the line element reads

$$
d s^{2}=-\left(1-w^{2}(r)\right) d v^{2}+2 d v d r .
$$

We shall not consider specific functions for $w^{2}$, as e.g. $2 M / r$ which describes a Schwarzschild black hole. Hence the coordinate $r$ should not be conceived as associated with spherical symmetry. In fact, from a 2D point of view, $r$ in Eq. (9) is an affine coordinate along $v=c s t$. We also notice that $r$ is affine along $\tau=$ cst., where $\tau$ is the proper time appearing in Painlevé-Gullstrand (PG) coordinates [13] where $d s^{2}=-d \tau^{2}+(d r-w d \tau)^{2}$. This guarantees that the forthcoming analysis can be made in PG coordinates.

When $w$ is a constant, the geometry is Minkowski. Therefore the differences with Sec. II will all stem from the gradient of $w(r)$. We have adopted these coordinates because they are well behaved in the late time portion of the space-time which is relevant for the Hawking effect and the correlations across the horizon, see e.g. [5]. In particular they stay regular when $w^{2}$ crosses 1 , say at $r=r_{h}$. This locus is the future horizon (for the static observers at fixed $r>r_{h}$ ) and it is a null line $U=c s t$. The absence of the corresponding $V$ (past) horizon induces a disymmetry: left moving modes are regular across the horizon $U=c s t$. and play no role in the following.

Assuming that $w(r) \rightarrow w_{\infty}=c s t$. for $r \rightarrow \infty$, the asymptotic observers at fixed $r$ are inertial, at rest with the black hole, and their proper time is $\tau_{\text {as }}=v\left(1-w_{\infty}^{2}\right)^{1 / 2}$. By rescaling $v$ and $r$, one can work in a gauge in which $w_{\infty}=0$. From now on we adopt it. The positive norm modes that these observers will use are $\phi_{\omega}^{\text {as }}=e^{-i \omega u} /(4 \pi \omega)^{1 / 2}$, with $\omega>0$. When $r \rightarrow \infty, u, v$ are related to $\tau_{a s}$ by $\tau_{a s}=(u+v) / 2$. At finite $r$, one has $u=v-2 \int d r /\left(1-w^{2}\right)$. One sees that $u$ might diverge when $w^{2}$ crosses 1 .

To settle this, one needs to know how $w$ behaves across $r_{h}$. We assume a regular behavior $w^{2}(r) \sim 1-2 \kappa\left(r-r_{h}\right)$ as this is the case for regular collapses. $\left(\kappa>0\right.$ coincides with the surface gravity since $w_{\infty}=0$ [30]. The extreme case $\kappa=0$ requires a special treatment [36].) At fixed $v$, one finds $u \sim-\kappa \ln \left(r-r_{h}\right)$. Therefore, when $r \rightarrow r_{h}^{+}$at fixed $v$, $\phi_{\omega}^{\text {as }}$ behaves as

$$
\phi_{\omega}^{\mathrm{as}}(u) \sim \frac{\left(r_{v}(u)-r_{h}\right)^{i \omega / \kappa}}{(4 \pi \omega)^{1 / 2}}
$$

3 The connected $2 \mathrm{pt}$ function of $T_{\mu \nu}$ governs gravitational back-reaction effects beyond those included in the semi-classical Einstein equations. 22] This provides its dynamical relevance. 
and can be taken to vanish for $r<r_{h}$. The function $r_{v}(u)$ obeys

$$
d r_{v}=-\frac{1}{2} C(u, v) d u
$$

where $C(u, v)=\left(1-w^{2}\right)$ is the conformal factor in the $u, v$ coordinates. It should be emphasized that $r_{v}(u)$ is an affine parameter for all $w(r)$, and this property is governed by $C$ which appears here for the second time. (It was implicitly used before when imposing that the coordinates $u, v$ are related to the asymptotic proper time by $2 \tau_{a s}=u+v$.)

When replacing the acceleration $a$ by $\kappa$ in Eq. (10), the correspondance with Eq. (A2) is manifest, and physically meaningful because $r_{v}$ is affine, as $U$ is in Minkowski space. However, this correspondence is confined near the horizon since $\phi_{\omega}^{\text {as }}$ behaves as $e^{i 2 \omega r}$ for $r \rightarrow \infty$ at fixed $v$. Therefore, the gradual change of the mode $\phi_{\omega}$ from $\sim\left(r-r_{h}\right)^{i \omega / \kappa}$ to $\sim e^{i 2 \omega r}$ is what distinguishes the back hole case from Minkowski. These facts have been recognized in [1, 24] and shall be further exploited below.

The second part of the correspondence concerns the state of $\phi$. While in Minkowski space the notion of vacuum is unambiguous, in a black hole geometry this is lost. Nevertheless the late time behavior [8] is universal and stationary when described in terms of $u$ (or $\tau_{\mathrm{as}}$ ). This can be verified by considering different collapses and different initial states: in each case there are transients, but after a few e-folding $u$-times $1 / \kappa$, these fade out as $\sim e^{-\kappa u}$ and the stationary values set in (unless the collapse and/or the state is singular). Therefore, as far as the description in terms of $u$ is concerned, a single stationary state is selected. As noticed in [1], this state is most simply characterized by Unruh modes, $\phi_{\omega}$, exactly as the Minkowski vacuum can also be, see the discussion after Eq. A2). The particular combination of modes weighted by $z_{\omega}=e^{-\pi \omega / \kappa}$ means that $\phi_{\omega}$ only contains positive frequencies $\Omega_{K}=i \partial_{U_{K}}$ where $U_{K}$ is the regular coordinate across $r_{h}$ which is related to $u$ by $d U_{K} / d u=e^{-\kappa u}$. Indeed, stationarity requires an exponential relation bewteen $u$ and $U_{K}$, whereas regularity across $r_{h}$ fixes the decay rate $d \ln U_{K} / d u$ to be $\kappa$.

The correspondence is now completed: the regularity and the stationarity of the state allow to characterize it by the modes of Eq. A2 , with $U$ replaced by $U_{K}=-e^{-\kappa u} / \kappa$. This exponential is no longer related to a Lorentz transformation, but is still related to an isometry whose Killing field norm $\propto\left(1-w^{2}\right)^{1 / 2}$ vanishes on the horizon.

\section{Correlation functions and asymptotic quanta}

In the Unruh vacuum, the connected 2pt function of $T_{U U}=\left(\partial_{U_{K}} \phi\right)^{2}$, see Eq. (7), is exactly given by Eq. (1). Then, since $\kappa u=-\ln \left(-\kappa U_{K}\right)$, Eq. (2) with $a \rightarrow \kappa$ also obtains. However, as discussed in III. A, there is nothing special about these identities. What makes Eq. (2) meaningfull here is that both $U_{K}$ and $u$ are affine, respectively across the horizon and asymptotically. Hence Eq. (2) tells us that when evaluated in the regular state, the 2pt function used by inertial asymptotic observers is that of a thermal flux at the Hawking temperature $\kappa / 2 \pi[3]$.

Let us now consider the equivalent of Eq. (3). Introducing on the other side of the horizon $\kappa \bar{u}=\ln \kappa U_{K}$, Eq. (3) with $a \rightarrow \kappa$ automatically obtains. But what does it mean? Unlike for the Unruh effect, we cannot refer to accelerated systems in the $L$ quadrant. Nevertheless the procedure of [7] applies to black holes, and one can study the $T_{\mu \nu}$ correlated to the detection of an asymptotic quantum. As in Minkowski, there is a reduction of the state: expectation values should be computed with the reduced density matrix, see App. C. Then, because of the entanglement of Eq. (B2), there is a correlation between $T_{\mu \nu}$ evaluated inside the horizon and a detection on $\mathcal{I}^{+}$[10]. Equivalently, one can directly look for correlations in energy across the horizon and obtain Eq. (3).

There is however an important difference between Eq. (2) and Eq. (3). It originates from the different status of the coordinates $u$ and $\bar{u}$. Eq. (2) has a clear interpretation because $d u$ at fixed $v$ is affine for $r \rightarrow \infty$. If we can consider the equivalent of Eq. (2) with both points inside, and obtain the same answer when using $\bar{u}$, we should ask under which conditions would $\left\langle T_{u u}^{L} T_{u u}^{L}\right\rangle$ posses an intrinsic meaning (without any reference to the external region). For this, it "suffices" that the inside region be also infinite and that $w \rightarrow$ const. for $r \rightarrow-\infty$. Whereas it is unlikely that this be relevant for black holes, we assume it is the case and consider the consequences. (In a next Section we discusss analogue black holes where this possibility can easily be realized.) When $w \rightarrow$ const. for $r \rightarrow-\infty$, the notion of asymptotic quanta equally applies to the negative frequency partners [35]. Then, $\left\langle T_{u u}^{L} T_{u u}^{L}\right\rangle$ has the same meaning as $\left\langle T_{u u}^{R} T_{u u}^{R}\right\rangle$, and the long distance correlation $\left\langle T_{u u}^{L} T_{u u}^{R}\right\rangle$ of Eq. (3) can be probed.

\section{Locality, covariance and renormalization}

To interprete Eq. (7) we have so far used the asymptotic properties of the metric. However we would like an intrinsic description of the gradual transition from the horizon to the asymptotic regions. To this end we consider the renormalization procedure. As we shall see its covariance supplies the intrinsic description we are looking for, 
and this by breaking the conformal invariance. (It should be clear that different rules on how to interpolate will give different behaviors since the $2 \mathrm{pt}$ function of $T_{U U}$ is not a scalar).

We noticed that both $u$ and $U_{K}$ are affine respectively for $r \rightarrow \infty$ and $r \sim r_{h}$. We also noticed that $r_{v}$ of Eq. (11) is affine all the way through. In what follows we exploit this to relate the 2 pt function of $T_{U U}$ to its renormalized value of Eq. (8). To this end, we study the coincidence point limit of Eq. (7) in the Unruh vacuum using

$$
T_{r r}=\left(\partial_{r} \phi\right)^{2}=\left(\frac{d U}{d r}\right)^{2} T_{U U}
$$

which is "coordinate invariant" since $r_{v}$ is, up to a scale, globally defined. To present the concepts we first work at spatial infinity. There, in the limit $u_{0} \rightarrow u$, one has

$$
\begin{aligned}
\left\langle T_{u u}(u) T_{u u}\left(u_{0}\right)\right\rangle_{K} & =\left(\frac{d U_{K}(u)}{d u} \frac{d U_{K}\left(u_{0}\right)}{d u_{0}}\right)^{2}\left(\frac{1}{4 \pi} \frac{1}{\left(U_{K}(u)-U_{K}\left(u_{0}\right)-i \epsilon\right)^{2}}\right)^{2} \\
& =\left(\frac{1}{4 \pi} \frac{1}{\left(u-u_{0}-i \epsilon\right)^{2}}\right)^{2}+\frac{2\left\langle T_{u u}(u)\right\rangle_{K}^{\mathrm{ren}}}{4 \pi\left(u-u_{0}-i \epsilon\right)^{2}}+O\left(\frac{1}{u-u_{0}}\right),
\end{aligned}
$$

where $\left\langle T_{u u}(u)\right\rangle_{K}^{\text {ren }}$ is the asymptotic expectation value of $T_{u u}$ in the Unruh vacuum. Indeed, it is defined [5, 9] as

$$
\begin{aligned}
\left\langle T_{u u}(u)\right\rangle_{K}^{\mathrm{ren}} & =\lim _{u_{0} \rightarrow u} \partial_{u} \partial_{u_{0}}\left(\left\langle\phi(u) \phi\left(u_{0}\right)\right\rangle_{K}-\left\langle\phi(u) \phi\left(u_{0}\right)\right\rangle_{M}\right) \\
& =\frac{1}{12 \pi}\left(\frac{d U_{K}}{d u}\right)^{1 / 2} \partial_{u}^{2}\left(\frac{d U_{K}}{d u}\right)^{-1 / 2}=\frac{\kappa^{2}}{48 \pi},
\end{aligned}
$$

where $\left\langle\phi(u) \phi\left(u_{0}\right)\right\rangle_{M}=\frac{-1}{4 \pi} \ln \left(u-u_{0}-i \epsilon\right)$ is evaluated in the asymptotic (Boulware) Minkowski-like vacuum. The procedure of Eq. (13) is clear: by subtracting the asymptotic vacuum divergence, one extracts the excess in the "noise" and thus identifies the mean value of Eq. (14). Moreover, this procedure can be univocally covariantized and applied at every space time point. This is achieved by making use of $r_{v}(u)$ :

$$
\begin{aligned}
\left\langle T_{r r}(u) T_{r r}\left(u_{0}\right)\right\rangle_{K}= & \left(\frac{1}{4 \pi} \frac{1}{\left(r_{v}(u)-r_{v}\left(u_{0}\right)+i \epsilon\right)^{2}}\right)^{2} \\
& +2\left\langle T_{r r}(u, v)\right\rangle_{K}^{\mathrm{ren}}\left(\frac{1}{4 \pi} \frac{1}{\left(r_{v}(u)-r_{v}\left(u_{0}\right)+i \epsilon\right)^{2}}\right)+\ldots
\end{aligned}
$$

where $\left\langle T_{r r}(u, v)\right\rangle_{K}^{\text {ren }}$ is the renormalized value of $T_{r r}$ which is defined as

$$
\begin{aligned}
\left\langle T_{r r}(u, v)\right\rangle_{K}^{\text {ren }} & =\lim _{u_{0} \rightarrow u} \partial_{r} \partial_{r_{0}}\left(\left\langle\phi(u) \phi\left(u_{0}\right)\right\rangle_{K}-\left\langle\phi(u) \phi\left(u_{0}\right)\right\rangle_{\text {local }}\right) \\
& =\frac{1}{12 \pi}\left(\frac{d U_{K}}{d r}\right)^{1 / 2} \partial_{r}^{2}\left(\frac{d U_{K}}{d r}\right)^{-1 / 2},
\end{aligned}
$$

where

$$
\left\langle\phi(u) \phi\left(u_{0}\right)\right\rangle_{\text {local }}=-\frac{1}{4 \pi} \ln \left(r_{v}(u)-r_{v}\left(u_{0}\right)+i \epsilon\right) .
$$

In virtue of the covariance, when using the affine coordinate $r_{v}$, the subtraction term possesses this universal form. This explains why Eq. (16) generalizes Eq. (14) at every point.

Several remarks should be made. Firstly, using Eq. (11), one verifies that Eq. (16) gives back Eq. (8) in all spacetimes and all vacua. Hence Eq. (16) can be seen as an alternative expression for it. Secondly, since the vacuum is defined through the coordinate $U_{K}$, and the subtraction only refers to the metric through Eq. (11), $\left\langle T_{r r}\right\rangle_{K}^{\text {ren }}$ is governed by $d U_{K} / d r$ and nothing else. Thirdly, even though $\left\langle T_{U U} T_{U U}\right\rangle_{K}$ is independent of $v$, the covariance of the divergent terms in Eq. (15) unambiguously defines the $v$-dependence of $\left\langle T_{r r}^{\mathrm{ren}}\right\rangle_{K}$ in Eq. (16). 


\section{E. Covariant description of stress tensor correlations}

Following the same logic, we use $T_{r r}$ of Eq. (12) to characterize the stress-energy correlations in the Unruh vacuum at every point. Using Eq. (1), Eq. (7) gives

$$
\begin{aligned}
\left\langle T_{r r}(v, r) T_{r r}\left(v_{0}, r_{0}\right)\right\rangle_{K} & =\left(\partial_{r} \partial_{r_{0}}\left\langle\phi(v, r) \phi\left(v_{0}, r_{0}\right)\right\rangle_{K}\right)^{2} \\
& =\left(\frac{d U_{K}}{d r} \frac{d U_{K}}{d r_{0}}\right)^{2}\left(\frac{1}{4 \pi} \frac{1}{\left(U_{K}(v, r)-U_{K}\left(v_{0}, r_{0}\right)-i \epsilon\right)^{2}}\right)^{2} .
\end{aligned}
$$

This bi-tensor field depends on $\Delta U_{K}^{-4}$ since the state is the Unruh vacuum, but unlike Eq. (1), it depends on the actual location of the two points through the $v$-dependence of the Jacobians $d U_{K} / d r$. Using $U_{K}(v, r)=e^{-\kappa v} \mathcal{U}_{K}(r)$ which follows from the stationarity of the metric, we can extract this $v$-dependence and obtain

$$
\left\langle T_{r r}(v, r) T_{r r}\left(v_{0}, r_{0}\right)\right\rangle_{K}=\left(\frac{d \mathcal{U}_{K}}{d r} \frac{d \mathcal{U}_{K}}{d r_{0}}\right)^{2}\left(4 \pi\left(e^{\frac{-\kappa\left(v-v_{0}\right)}{2}} \mathcal{U}_{K}-e^{\frac{\kappa\left(v-v_{0}\right)}{2}} \mathcal{U}_{K}^{0}-i \epsilon\right)^{2}\right)^{-2}
$$

There is yet another interesting way to write this correlator. Using

$$
1-w^{2}=2 \kappa\left(\frac{d \ln \mathcal{U}_{K}}{d x}\right)^{-1}
$$

which follows from Eq. (11), we get

$$
\left\langle T_{r r}(v, r) T_{r r}\left(v_{0}, r_{0}\right)\right\rangle_{K}=\left(\frac{\kappa^{2}}{\pi} \frac{U_{K} U_{K}^{0}}{\left(1-w^{2}\right)\left(1-w_{0}^{2}\right)} \frac{1}{\left(U_{K}-U_{K}^{0}-i \epsilon\right)^{2}}\right)^{2} .
$$

From Eq. (19), it is clear that the correlator is a function of only 3 variables, $r, r_{0}$ and $v-v_{0}$, the state (Unruh vacuum) being stationary. Because it still depends on 3 variables, its behavior in different 2 dimensional sections illustrates different aspects of the correlations associated with the Hawking effect. When fixing $r_{0}, v_{0}$ on $\mathcal{I}^{+}$, Eq. (18), function of $r, v$, describes the correlations associated with a late detection on $\mathcal{I}^{+}\left[10\right.$. Instead, at equal time $v_{0}=v$, Eq. (18) describes the correlations in the $r, r_{0}$ plan that have been accumulated in the past of that time [19].

Before studying these two cases, we need to be more precise about the black hole geometries we shall work with. As explained before, we consider profiles that become constant for $r \rightarrow \pm \infty$. To have a simple example at hand, we choose directly $\mathcal{U}_{K}(r)$ since it is the only relevant function in Eq. (19):

$$
\kappa \mathcal{U}_{K}(x)=-\left(e^{2 \kappa x}-e^{-2 \bar{\kappa} x}\right)
$$

where $x=r-r_{h}$. This is a kind of symmetrized version of the Schwarzschild metric where $\mathcal{U}_{K}=-x e^{2 \kappa x}$. Using Eq. (20), one gets

$$
1-w^{2}=\frac{e^{2 \kappa x}-e^{-2 \bar{\kappa} x}}{e^{2 \kappa x}+\frac{\bar{\kappa}}{\kappa} e^{-2 \bar{\kappa} x}} .
$$

Near the horizon, for $\kappa x \ll 1$, one has $w^{2}=1-2 \kappa x$ for all values of $\bar{\kappa}$, and the asymptotic values are $w_{\infty}=0$ for $x \rightarrow \infty$, and $w_{-\infty}^{2}=1+\bar{\kappa} / \kappa$ for $x \rightarrow-\infty$.

\section{Correlations to a late detection}

We fix $x_{0}, v_{0}$ on $\mathcal{I}_{R}^{+}$, on the future right infinity, and label it with $u_{0}$. We consider Eq. (18)

$$
\left.\bar{T}_{r r}(r, v)\right|_{u_{0}, \mathcal{I}+} \equiv\left\langle T_{r r}(v, r) T_{r r}\left(u_{0}, \mathcal{I}_{R}^{+}\right)\right\rangle_{K}
$$

as a 1 pt function. As such $\bar{T}_{r r}(r, v)$ is a special case of the conditional value

$$
\left.\bar{T}_{r r}(r, v)\right|_{\Pi_{R}}=\left\langle T_{r r}(v, r) \hat{\Pi}_{\mathcal{I}_{R}^{+}}\right\rangle_{K}
$$




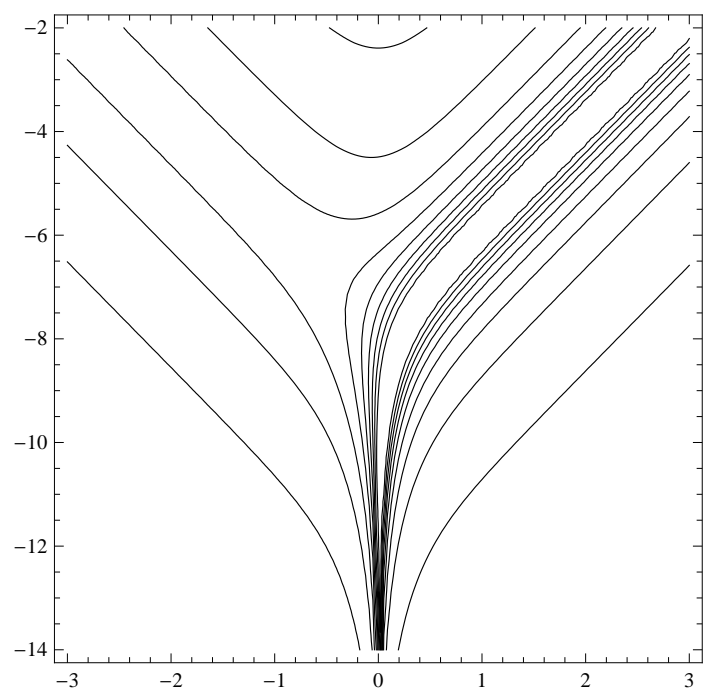

Figure 1: The stress-energy conditional to a late detection. We represent $\bar{T}_{r r}$ of Eq. (24) in the $x, v$ plane ( $v$ being vertical) for a detection at $x_{0}=4, v_{0}=0$, outside the picture in the top right-hand region where $w(r)$ is constant. The horizon is at $x=0$ and $\kappa=\bar{\kappa}=1$ in Eq. (23). When $|x| \geq 1$, on both sides, the pattern is translation invariant along the null direction because $w$ is constant. Instead for $|x|<1$, one sees the endless focusing of the null lines for $v \rightarrow-\infty$. The "post-selected" partner propagates along $v+2 x=-8=v_{0}-2 x_{0}$, i.e. along the opposite trajectory fixed by $x_{0}, v_{0}$. These features were found in [10] considering Eq. (25) for a typical Hawking quantum.

where $\hat{\Pi}_{\mathcal{I}_{R}^{+}}$is a projector that specifies a state on $\mathcal{I}_{R}^{+}$, see App. C for a brief account, and App. C of [5] for details. The projector can be chosen at will. If it is taken to be $a_{\omega}^{a s \dagger} a_{\omega}^{a s}$ it selects the subset of states (present in the Unruh) which contains that asymptotic quantum without specifying when it is detected. One can also consider the other limit in which one only specifies the moment of detection $u_{0}$. In that case, Eq. (25) is identical to Eq. (24), up to an overal constant factor, as can be seen by taking $f(\tau)=\delta\left(\tau-u_{0}\right)$ in Eqs. $(55,56)$ of $[10]$. From this we learn the physical meaning of Eq. (24): it gives the mean value of $T_{r r}$ when the in state is Unruh vacuum, and when a particle is detected on $\mathcal{I}^{+}$at $u=u_{0}$.

When $(r, v)$ is also on $\mathcal{I}_{R}^{+}$and coordinated by $u$, Eq. (24) is given by Eq. (2) (times $4^{2}$ ), as can be seen using Eq. (21), and $\kappa u=-\ln \left(-\kappa U_{K}\right)$. In this we recover the thermal correlations [3] of the asymptotic radiation. When $(r, v)$ is taken on the opposite null infinity $\mathcal{I}_{L}^{+}$, or sufficiently far away from the horizon so that $w$ is constant, $\bar{T}_{r r}$ behaves as Eq. (3) when using the mirror coordinate $\kappa \bar{u}=\ln \left(\kappa U_{K}\right)$. Moreover, when $(r, v)$ is near the horizon, $\kappa\left(r-r_{h}\right) \ll 1$, and parameterized by $U_{K}, \bar{T}_{r r}$ behaves as in Eq. (1), as can be seen using Eq. (18) and $d U_{K} / d r \sim-2$ for $r=r_{h}$. In this we recover that when probed near the horizon, for $\kappa x \ll 1$, Unruh vacuum behaves like Minkowski vacuum.

In addition to these three asymptotic behaviors, the non-trivial information contained in $\bar{T}_{r r}$ of Eq. (24) is the smooth interpolation from one to the other, which is represented in Fig. 1. From this we clearly see the gradual emergence from $v=-8$ of the energy flux associated with the partner on the other side of the horizon. What is nontrivial is the following. In the past of that time, $\bar{T}_{r r}$ is essentially constant along the outgoing null lines $U_{K}=$ const. and, as could have been expected, behaves exactly as $\bar{T}_{U U}$ would in Minkowski. On the contrary, near the horizon and in the future, $\bar{T}_{r r}$ behaves very differently since the lines $\bar{T}_{r r}=$ const. cross the horizon. This peculiar behavior could not have be found had we studied the 2pt function of Eq. (7) because the latter obeys Eq. (11) and depends only on $U$, even in a black hole geometry. This establishes that the use of $T_{r r}$ of Eq. (12) with $r$ affine is truly necessary. It should be also noticed that the above behavior of $\bar{T}_{r r}$ cannot be found in Minkowski using affine coordinates either. In fact, it is characteristic of pair creation processes, as can be seen by comparing Fig.1 to Fig. 1.1 of [5] which describes pair creation in an electric field. As noticed in [10], these properties of $\bar{T}_{r r}$ provide a clear answer to the long standing question: where is a Hawking quantum "born"? [25].

\section{Equal time correlations}

We consider Eq. (18) at equal EF time $v=v_{0}$. Since $U_{K}=e^{-\kappa v} \mathcal{U}_{K}$, the correlator

$$
C_{K}\left(r, r_{0}\right)=\left\langle T_{r r}(r) T_{r r}\left(r_{0}\right)\right\rangle_{K}=\left(\partial_{r} \partial_{r_{0}}\left\langle\phi(r) \phi\left(r_{0}\right)\right\rangle_{K}\right)^{2},
$$



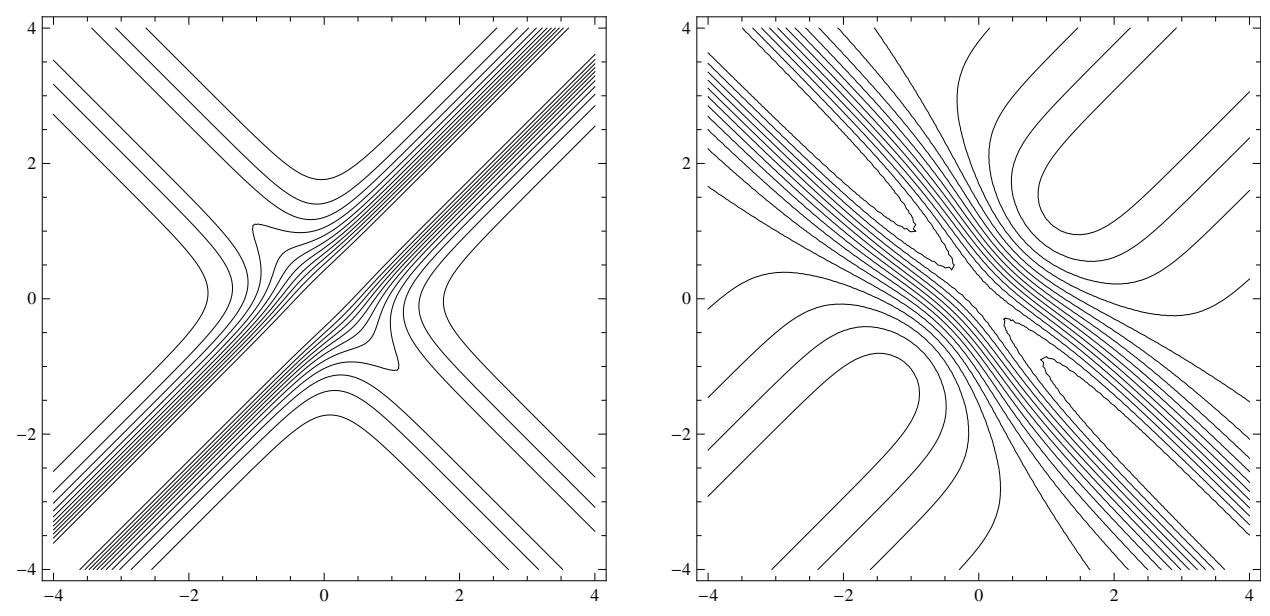

Figure 2: Equal time correlations. On the left, we represent Eq. (26), and on the right, Eq. (29), both in the metric of Fig. 1. The horizon is at $x, x_{0}=0$. On the left, the signal diverges for $x \rightarrow x_{0}$ whereas the pattern along $x+x_{0}=0$ represents Eq. (28). It is translation invariant in $x-x_{0}$ once $w$ has reached a constant. On the right, the subtracted $S_{K}$ is everywhere finite and regular. It is dominated by the correlations across the horizon. The subdominant patterns centered along $x-x_{0}=0$ are due to the fact that the un-subtracted correlator in Eq. (29) decreases faster than the subtraction. On the diagonal, $S_{K}(x, x)=\left\langle T_{r r}(x)\right\rangle_{K}^{\text {ren }}$, the renormalized flux of Eq. (16).

is given by Eq. (21) with $U_{K}$ replaced by $\mathcal{U}_{K}$. Using $\mathcal{U}_{K}$ of Eq. (22), $C_{K}\left(r, r_{0}\right)$ diverges as $\sim\left(r-r_{0}\right)^{-4}$ when $r \rightarrow r_{0}$, as expected since the Unruh vacuum is a Hadamard state. Moreover, when $r \rightarrow \infty$, since $\mathcal{U}_{K} \sim-e^{-2 \kappa r}$, one has

$$
C_{K}\left(r, r_{0}\right)=\frac{\kappa^{4}}{16 \pi^{2} \sinh ^{4}\left(\kappa\left(r-r_{0}\right)\right)} .
$$

The thermal noise of Eq. (2) associated with the asymptotic radiation is properly encoded in $C_{K}$ since at fixed $v$, $d u=-2 d r$. Similarly, when $x$ and $x_{0}$ are on opposite sides of the horizon, since $\mathcal{U}_{K} \sim-e^{-2 \bar{k}|x|}$ for $x \rightarrow-\infty$, one asymptotically gets

$$
C_{K}\left(r, r_{0}\right)=\frac{\kappa^{2} \bar{\kappa}^{2}}{\left.16 \pi^{2} \cosh ^{4}\left(\bar{\kappa} x+\kappa x_{0}\right)\right)},
$$

thereby recovering Eq. (3), and making contact with [18, 19], see Fig. 2 on the left. To further investigate the gradual change from near horizon configurations to long distance correlations, we consider the subtracted $2 \mathrm{pt}$ function ${ }^{4}$

$$
S_{K}\left(r, r_{0}\right)=\partial_{r} \partial_{r_{0}}\left[\left\langle\phi(r) \phi\left(r_{0}\right)\right\rangle_{K}-\frac{(-1)}{4 \pi} \ln \left(r-r_{0}\right)\right] .
$$

The substraction term is the same as in Eq. (17), but considered here for all values of $r-r_{0}$. From the the right plot in Fig. 2, we see that the residual signal is free of UV divergences and describes the emergence of the pairs on distances $\kappa\left(r-r_{h}\right)=\kappa x \sim 1$, i.e. characterized by the geometry.

One first notices that the correlations across the horizon in $S_{K}$ are negative (as in inflation [26]). In fact the correlator $\left\langle\partial_{r} \phi \partial_{r_{0}} \phi\right\rangle_{K}$ is negative "everywhere", as can be seen from Eq. (19) and Eq. (22). We have added quotation marks because this correlator is a distribution. In fact, the coincidence point limit is ruled by the $i \epsilon$, see Eq. (11). It specifies that (the real part of) $\left\langle\partial_{r} \phi \partial_{r_{0}} \phi\right\rangle_{K}$ diverges positively for $r \rightarrow r_{0}$ same $v$, and it ensures that the integral $\int_{-\infty}^{\infty} d U\left\langle\partial_{U} \phi \partial_{U_{0}} \phi\right\rangle$ vanishes in the Minkowski vacuum. (This is reminiscent of the behavior of $\left\langle T_{U U}\right\rangle_{F R}$, the mean flux evaluated in the Fulling-Rindler vacuum [29].) We also notice that for close points, $S_{K}$ is positive because the subtraction is larger than the "bare" term. Moreover when evaluated at the same point, $S_{K}(x, x)$ is equal to $\left\langle T_{r r}(x)\right\rangle_{K}^{\text {ren }}$ of Eq. (16). For $x \rightarrow \infty$ one verifies that $S_{K}(x, x)=(-1 / 3) \times S_{K}(x,-x)$. Finally we notice that Fig.2. is symmetrical under $x \rightarrow-x$. This follows from the symmetry of $1-w^{2}$ in Eq. (23) when $\kappa=\bar{\kappa}$. When $\bar{\kappa} / \kappa \gg 1$, this is lost, and $1-w^{2}$ resembles more to Schwarzschild. We hope to report on this case soon.

\footnotetext{
${ }^{4}$ For simplicity we worked with the square root of Eq. (26) rather than $C_{K}$ itself. The reason is that the subtraction needed to obtain a finite expression for $r \rightarrow r_{0}$ is more complicated, as it requires three terms. The subtracted correlator of $\left\langle T_{r r} T_{r r}\right\rangle_{K}$ possesses the same behavior as $S_{K}$.
} 


\section{F. Time-dependent growth of flux and correlations}

So far we considered the stationary correlation patterns found in the Unruh vacuum. We now consider the early transient effects. As already said in Sec. III.B, they are not universal. However their late time behavior is when the state contains no high frequency excitations, i.e. when it is an Hadamard state. There is a simple and efficient way to characterize this behavior. It consists in assuming that the initial state specified at $v=v_{\text {in }}$ is the Minkowski vacuum. (This state can be found in a gravitational collapse when the infalling matter is a light-like thin shell [10].) In this state, $v=v_{\text {in }}$, the 2 pt function at is $G^{\text {in }}\left(r, r_{0}\right)=\frac{-1}{4 \pi} \ln \left(r-r_{0}+i \epsilon\right)$, as in Eq. (17). In the future, one has

$$
G^{\text {in }}\left(r, v ; r_{0}, v_{0}\right)=-\frac{1}{4 \pi} \ln \left(X^{\text {in }}(r, v)-X^{\text {in }}\left(r_{0}, v_{0}\right)+i \epsilon\right)
$$

where $X^{\text {in }}(r, v)$ gives the value of $x=r-r_{h}$ hit by the outgoing null ray issued from $r, v$ when it crosses $v_{\text {in }}$. In this state, the mean flux of Eq. (16) becomes

$$
\left\langle T_{r r}(r, v)\right\rangle_{\text {in }}^{\text {ren }}=\frac{1}{12 \pi}\left(\frac{d X^{\text {in }}(r, v)}{d r}\right)^{1 / 2} \partial_{r}^{2}\left(\frac{d X^{\text {in }}(r, v)}{d r}\right)^{-1 / 2}
$$

and the correlator of Eq. (18) is

$$
\left\langle T_{r r}(v, r) T_{r r}\left(v_{0}, r_{0}\right)\right\rangle_{\text {in }}=\left(\frac{d X^{\text {in }}}{d r} \frac{d X^{\text {in }}}{d r_{0}}\right)^{2}\left(\frac{1}{4 \pi} \frac{1}{\left(X^{\text {in }}(v, r)-X^{\text {in }}\left(v_{0}, r_{0}\right)+i \epsilon\right)^{2}}\right)^{2}
$$

In both expressions one has simply replaced $U_{K}$, the Kruskal coordinate encoding the Unruh vacuum, by $X^{\text {in }}$ which encodes the time-dependent state which is vacuum at $v_{\text {in }}$.

In the metrics of Eq. (23), these expressions flow towards Eq. (16) and Eq. (18), exponentially in $v-v_{\text {in }}$ in the near horizon region where $\partial_{r} w=\kappa$, and linearly when $\partial_{r} w \sim 0$. To show this we make use of $\mathcal{U}_{K}(x)$, solution of Eq. (20). Calling $X\left(\mathcal{U}_{K}\right)$ the inverse function, and using $U_{K}(x, v)=\mathcal{U}_{K}(x) e^{\kappa v}$, we obtain

$$
X^{\mathrm{in}}(r, v)=X\left[\mathcal{U}_{K}(x) e^{-\kappa\left(v-v_{\mathrm{in}}\right)}\right]
$$

for all profiles $w^{2}(x)$. To give a simple example, we use Eq. (22) with $\bar{\kappa}=\kappa$, and we get

$$
\begin{aligned}
2 \kappa X^{\mathrm{in}}(r, v) & =\operatorname{arcsinh}\left(e^{-\kappa\left(v-v_{\text {in }}\right)} \sinh (2 \kappa x)\right), \\
\frac{d X^{\text {in }}}{d x} & =\frac{\cosh (2 \kappa x)}{\left[e^{2 \kappa\left(v-v_{\text {in }}\right)}+\sinh ^{2}(2 \kappa x)\right]^{1 / 2}} .
\end{aligned}
$$

In Fig. 3, on the left, we represent Eq. (32) evaluated at equal time for different values of $v-v_{\text {in }}$. In these plots, one clearly sees the growth of the correlations across the horizon with a rate given by $\kappa$ [19]. One also observes a narrowing of the spread of the dominant correlations centered along $x=x_{0}$. This is due to the progressive replacement of vacuum correlations $\sim 1 /\left(x-x_{0}\right)^{2}$ by the thermal ones $\sim \kappa^{2} / \sinh ^{2} \kappa\left(x-x_{0}\right)$ of Eq. (27). On the right plots, the subtracted function $S_{\text {in }}\left(r, r_{0}\right)$, the equivalent of Eq. (29) evaluated in the in vacuum, displays both the growth of the long distance correlations, and the modifications of the local correlations.

It is worth analyzing these time dependent effects through two other perspectives. In Fig. 4, on the left, we present the mean flux of Eq. (31) in the $x, v$ plane from the onset of the vacuum at $v=v_{\text {in }}=0$. $\left\langle T_{r r}\right\rangle_{\text {in }}$ contains transients which propagate along $u=v-2 x \sim 0$. It then reaches a constant $x$-dependent profile. For $x \rightarrow \infty$ one recovers the standard value $\kappa^{2} / 12 \pi[9]$. In the metric of Eq. (22) with $\kappa=\bar{\kappa},\left\langle T_{r r}\right\rangle_{\text {in }}$ crosses 0 for $\sinh 2 x=\sqrt{2}$, i.e. $x \sim \pm .57$, and on the horizon, it is negative and equal to $-2 \kappa^{2} / 12 \pi$. When including gravitational back-reaction effects, this term participates to the evaporation of the black hole, see [27] for a numerical analysis in similar settings. On the right plot, we show $\left.\bar{T}_{\bar{u} \bar{u}}(\bar{u})\right|_{u_{0}}$ of Eq. (24), the flux correlated to a late detection at $u_{0}$ on $\mathcal{I}_{R}^{+}$, that we evaluate on $\mathcal{I}_{L}^{+}$ and parameterize with $\bar{u}$, the mirror coordinate $\kappa \bar{u}=\ln \kappa U_{K}$. There is no correlations from $\mathcal{I}_{R}^{+}$to $\mathcal{I}_{L}^{+}$for $u_{0}<-1$ in conformity to the fact that no Hawking radiation as yet reached the null infinites. Then for positive $u_{0}$, the correlations settle to a stationary pattern centered around $u_{0}+\bar{u}=0$ found in the Unruh vacuum.

In conclusion it is interesting to observe that, even though the transients give rise to a higher value of the mean flux, they are not associated with stronger correlations across the horizon, and this because, unlike the steady Hawking 

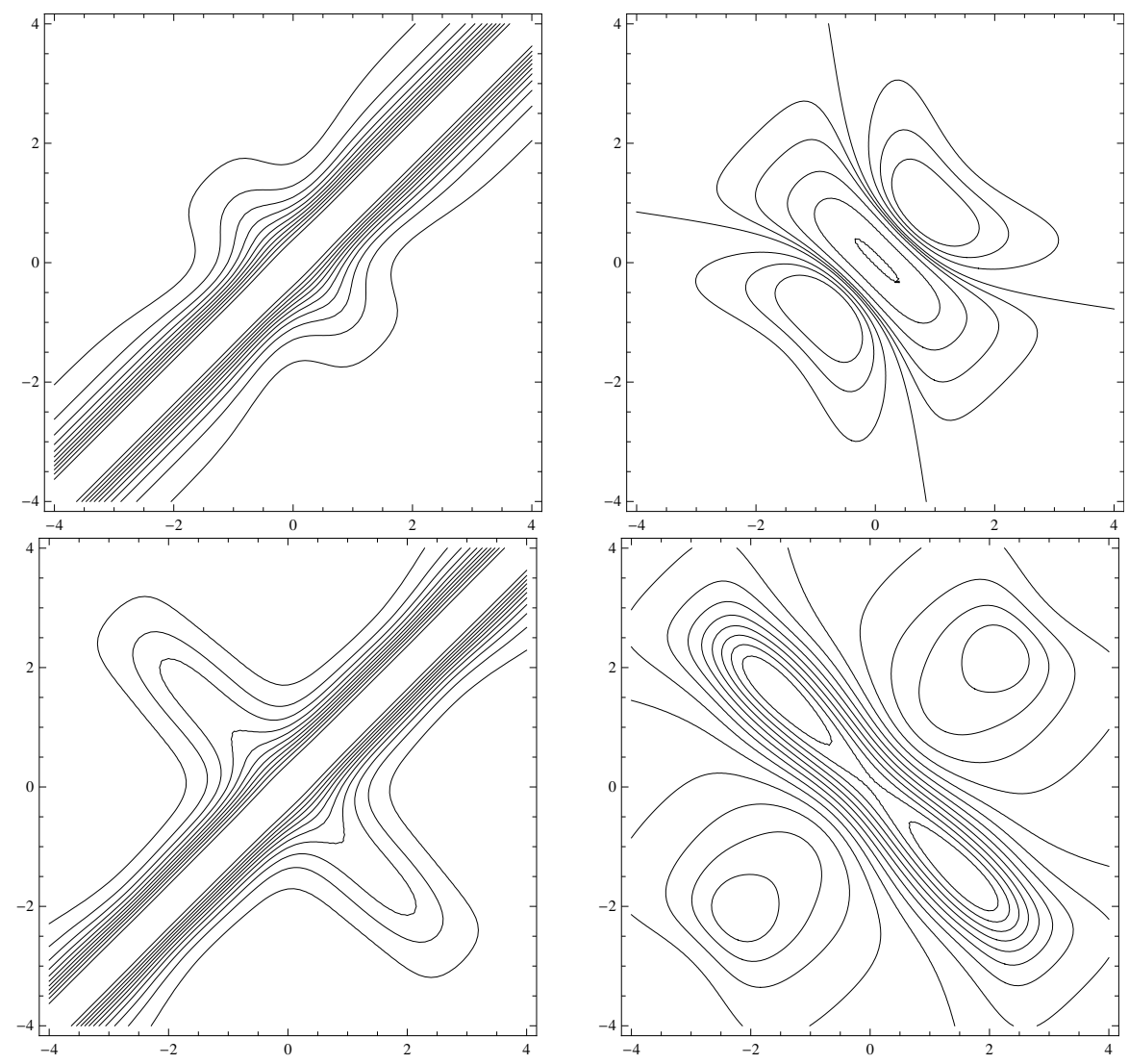

Figure 3: The growth of equal time correlations. We represent Eq. (32) at equal time on the left, and Eq. (29) on the right, after a lapse of time $\kappa\left(v-v_{\text {in }}\right)=1$ in the upper plots, and $\kappa\left(v-v_{\text {in }}\right)=4$ in the lower ones. On the left, one observes the growth of the correlations across the horizon centered along $x+x_{0}=0$. One also observes a narrowing of the correlations centered along $x=x_{0}$. On the right, $S_{\text {in }}\left(r, r_{0}\right)$ displays two distinct features. A strong signal associated with the building up of the correlations across the horizon, and subdominant patterns on both sides of the horizon due to the growth of thermal correlations of Eq. (27). At late times, both patterns asymptote to the stationary ones of Fig. 2.
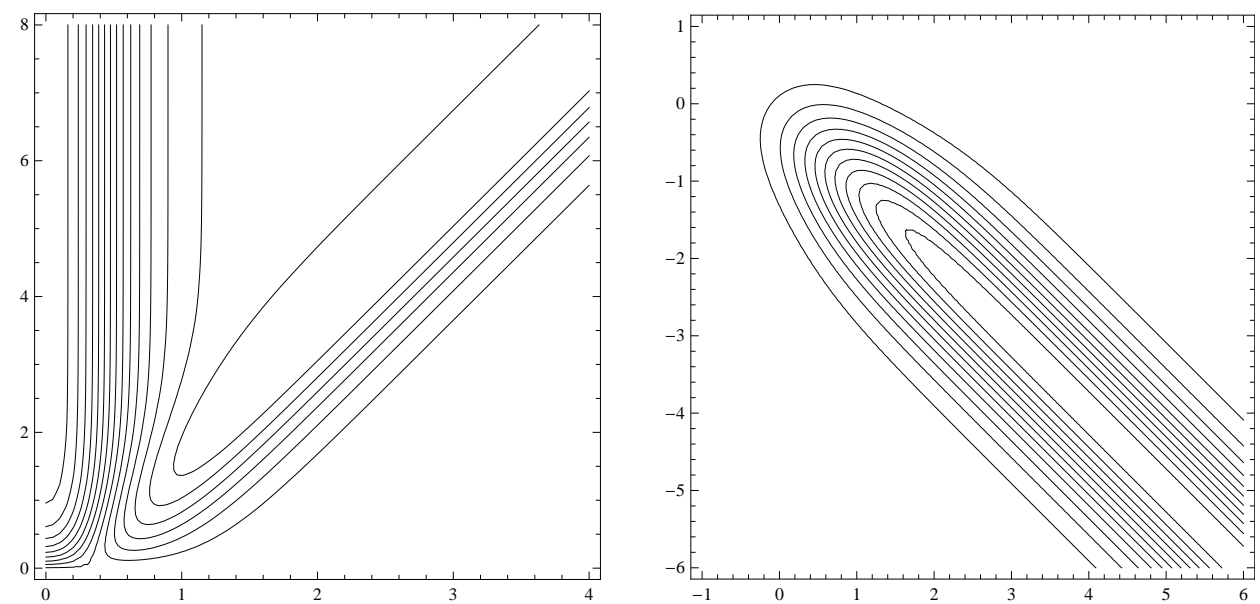

Figure 4: Time dependence of the mean flux and of the asymptotic correlations. On the left, we represent Eq. (31) with Eq. (34) from the onset of the vacuum at $v=0$ till $\kappa v=8$, and for $\kappa x$ from 0 to 4 (It is symmetric under $x \rightarrow-x$ ). The transients propagate on null lines $u=v-2 x \sim 0$. After they passed, $\left\langle T_{r r}\right\rangle_{\text {in }}$ is $v$-independent. On the right plot, we represent $\left.\bar{T}_{u u}(\bar{u})\right|_{u_{0}}$ of Eq. (24) in the $u_{0}, \bar{u}$ plane with $\bar{u}$ vertical, with $u_{0}$ and $\bar{u}$ defined on the future null infinity $v_{0}=v=\infty$. For $u_{0}<0$, before the transients, there is no correlations across the horizon. Instead for $u_{0}>3,\left.\bar{T}_{u u}(\bar{u})\right|_{u_{0}}$ only depends of $u_{0}+\bar{u}$, and is given by Eq. (3). 
radiation, the transients are not composed of entangled pairs of opposite frequency $\omega$. This can be checked by comparing $\left.\bar{T}_{u u}(\bar{u})\right|_{u}$ to the asymptotic flux $\left\langle T_{u u}\right\rangle_{\text {in }}$ evaluated for $x, v \rightarrow \infty$, with $u=v-2 x$ fixed:

$$
\begin{aligned}
\left\langle T_{u u}(u)\right\rangle_{\text {in }}^{\text {ren }} & =\frac{\kappa^{2}}{48 \pi} \frac{1+e^{-2 \kappa u}}{\left(1+e^{-2 \kappa u} / 4\right)^{2}} \\
\left.\bar{T}_{\bar{u} \bar{u}}(\bar{u})\right|_{u_{0}} & =\left(\frac{\kappa^{2}}{\pi}\right)^{2}\left(\left(e^{2 \kappa u_{0}}+1 / 4\right)\left(e^{-2 \kappa \bar{u}}+1 / 4\right)\left(\operatorname{arcsinh}\left(e^{-\kappa u_{0}} / 2\right)+\operatorname{arcsinh}\left(e^{+\kappa \bar{u}} / 2\right)\right)^{4}\right)^{-1} .
\end{aligned}
$$

\section{DISPERSIVE THEORIES AND ANALOGUE BLACK HOLES}

In a non-homogeneous medium, linear density fluctuations obey a relativistic d'Alembert equation in a curved metric when their wave lengths are larger than the inter-atomic distance [12]. Instead, for shorter wave lengths, the propagation becomes dispersive [28]. Assuming the speed of sound is constant and set to 1 , it can be described by

$$
\Omega^{2}=(\omega-w p)^{2}=F^{2}\left(p^{2}\right)
$$

where $\Omega$ is the co-moving frequency measured w.r.t. to the atoms, $w$ the velocity of the fluid, and $p, \omega$ the wave vector and the frequency measured in the lab. The dispersion can be incorporated in a modified field equation [11]

$$
\left(\partial_{\tau}+\partial_{x} w\right)\left(\partial_{\tau}+w \partial_{x}\right) \phi-\partial_{x}^{2} \phi \pm \frac{1}{\Lambda^{2}} \partial_{x}^{4} \phi=0
$$

which reduces to the relativistic one when sending the dispersive scale $\Lambda \rightarrow \infty$. For simplicity, we chose quartic, super $(+)$ or subluminal (-) dispersions: $F^{2}=p^{2} \pm p^{4} / \Lambda^{2}$.

Eq. (37) can then be used to study the impact of dispersion on Hawking radiation. In [11], the thermicity and the stationarity of the asymptotic radiation have been shown to be robust, i.e. hardly affected by dispersion when $\kappa \ll \Lambda$. This is sufficient for recovering Eq. (27). By constructing wave packets of in modes, it was then shown [16] that at large distance from the horizon one also recovers the correlations between Hawking quanta and their partners, and this, even though the early propagation was radically affected by dispersion. This second aspect is sufficient for obtaining Eq. (28), Fig. 2, and the late time properties of Fig. 1.

To clarify this, we shall compare the properties of wave packets of $\phi_{\omega}^{i n}$, the in modes of Eq. (37),

$$
\bar{\phi}_{\bar{\omega}}(\tau, x)=\int d \omega e^{-i \omega \tau} \phi_{\omega}^{i n}(x) \bar{f}_{\omega}
$$

where $\bar{f}_{\omega}$ selects the wave packet, with the correlation function

$$
\begin{aligned}
G^{i n}\left(\tau, x ; \tau_{0}, x_{0}\right) & \equiv\left\langle\phi(\tau, x) \phi\left(\tau_{0}, x_{0}\right)\right\rangle_{i n} \\
& =\int d \omega e^{-i \omega \tau} \phi_{\omega}^{i n}(x)\left[e^{-i \omega \tau_{0}} \phi_{\omega}^{i n}\left(x_{0}\right)\right]^{*},
\end{aligned}
$$

evaluated in the stationary in vacuum. When dispersion is weak, the similarity of expressions guarantees that similar patterns will be found. However, since dispersion grows as approaching the horizon, the way one probes the correlations, i.e. by extracting some limited range of $\omega$ through $\bar{f}_{\omega}$ in Eq. (38), or not as in Eq. (39), can lead to different behaviors. We now review the relevant points for achieving this comparison following [16, 30]; other treatments are mentioned in [31].

\section{A. Kinematics}

From a relativistic point of view, the presence of dispersion defines a preferred frame [14] which allows to define new scalars. This is best seen by "covariantizing" Eq. (36), i.e. by introducing a unit time-like vector field $u^{\mu}$, and viewing the field $\phi$ as propagating on a manifold endowed with both the metric and $u^{\mu}$. Then the energy in the preferred frame, and the spatial momentum perpendicular to it are respectively

$$
\Omega=u^{\mu} p_{\mu}, \quad p=s^{\mu} p_{\mu},
$$

where $-u^{2}=1=s^{2}$ and $s^{\mu} u_{\mu}=0$. The PG coordinates used in Eq. (37) can then be invariantly defined by $\partial_{x}=s^{\mu} \partial_{\mu}$ and $d \tau=u_{\mu} d x^{u}$. The field $u^{\mu}$ also defines the scalar density $\rho=u^{\mu} u^{\nu} T_{\mu \nu}$ which corresponds to the proper energy 
that observers following $d x^{\mu} / d \tau=u^{\mu}$ would measure. It is interesting to note that in the hydrodynamical limit one gets

$$
\rho=T_{x x}=\left(\partial_{x} \phi\right)^{2}
$$

In other words, $\rho$ coincides with $T_{r r}$ of Eq. (12) with $r$ defined by Eq. (11). This non-trivial correspondence follows from the affinity of $r$ at fixed $v$ and $\tau$, see the remarks after Eq. (9).

In the same spirit, we notice that the surface gravity measured with respect to the preferred frame is also scalar. It is given by the expansion [32] $\theta=u_{; \mu}^{\mu}=\partial_{x} w$ evaluated on the horizon. (The second expression is valid in PG coordinates). The ambiguity of the scale of the surface gravity in covariant theories is thus removed when using $u^{\mu}$.

\section{B. The modified modes}

In linear field theories, the modifications of the stress energy correlations due to dispersion will stem from the modifications of the modes, solutions of Eq. (37). When $\Lambda \gg \kappa$, these are modifications localized near the horizon, for $\kappa\left(r-r_{h}\right)=\kappa x \ll 1$. As noticed in [16] it is appropriate to work in the $p$-representation $\left(\right.$ with $\left.x=i \partial_{p}\right)$ with $w(x)$ linearized: $w=-1+\kappa x=-1+i \kappa \partial_{p}$. In this representation, Eq. (37) becomes

$$
(\omega-p w)(\omega-w p) \tilde{\phi}_{\omega}=F^{2} \tilde{\phi}_{\omega}
$$

Using $w=-1+i \kappa \partial_{p}$, the modified modes have the form, for details see the Appendix of [30],

$$
\tilde{\phi}_{\omega}=\tilde{\phi}_{\omega}^{0} \times e^{-i p / \kappa} \chi(p),
$$

where $\tilde{\phi}_{\omega}^{0}=|p|^{-i \omega / \kappa-1}$ is the standard dispersion less mode, and where $\chi$ obeys

$$
-\kappa^{2} \partial_{p}^{2} \chi=\frac{F^{2}}{p^{2}} \chi=H^{2} \chi
$$

The in state which generalizes the notion of the Unruh vacuum, the "free falling vacuum", is characterized by the positive norm modes which contain only positive $\Omega$ of Eq. (40). These in modes are related to the Unruh modes, see Eq. (A4),

$$
\tilde{\phi}_{\omega}^{U}=\theta(p) \frac{p^{-i \omega / \kappa-1}}{(4 \pi \kappa)^{1 / 2}}
$$

by $[16]$

$$
\tilde{\phi}_{\omega}^{i n}=\tilde{\phi}_{\omega}^{U} \times e^{-i p / \kappa} \chi(p),
$$

where $\chi(p)$ is the solution of Eq. (44) with a Wronskian equal to $\chi^{*} \partial_{p} \chi-\chi \partial_{p} \chi^{*}=2 i / \kappa$. The corresponding WKB solution is

$$
\chi(p)=\frac{1}{(H)^{1 / 2}} \exp \left(i \int_{p_{0}}^{p} H\left(p^{\prime}\right) d p^{\prime} / \kappa\right) .
$$

It provides a reliable approximation when $\Lambda \gg \kappa$. In the limit $\Lambda \rightarrow \infty$ fixed $p, H \rightarrow 1$ and $e^{-i p / \kappa} \chi \rightarrow 1$, thereby implying that $\tilde{\phi}_{\omega}^{i n}$ smoothly gives back $\tilde{\phi}_{\omega}^{U}{ }^{5}$

To understand the impact of dispersion, we now study the characteristics of Eq. (37) since the maximum of correlations will be localized along them. Having already the modes in $p$-space, the simplest way to get them is to consider Eq. (38) in $p$-space, and look for the stationary phase condition in $\omega$. Using Eq. (46), since $\chi$ is independent of $\omega$, one gets

$$
p(\tau)=p_{0} e^{-\kappa \tau}
$$

\footnotetext{
${ }^{5}$ At fixed $x$ instead, the limit $\Lambda \rightarrow \infty$ can be singular as some roots $p(x, \omega)$ of Eq. (36) are sent to infinity. The $p$-WKB approximation should not be confused with the usual one defined in $x$-space. For the Airy function, the modes in $p$ are exactly given by their $p$-WKB approximation. Similarly here, the corrections to Eq. (47) are negligible when $\Lambda / \kappa \gg 1$. This has been confirmed by numerical analysis, see [35] for a detailed study. From now on we neglect them and work in the adiabatic approximation with Eq. (47).
} 
irrespectively of the dispersion relation $F$, and thus as in relativistic theories.

To get the modified characteristics in $x$, we use $\omega-w p=F$, the root of Eq. (36) describing the right moving modes. Using $w \sim-1+\kappa x$, one gets [33]

$$
\begin{aligned}
& \frac{\kappa}{2}\left[x_{\omega}(p)-x_{-\omega}(p)\right]=\frac{\omega}{p}, \\
& \frac{\kappa}{2}\left[x_{\omega}(p)+x_{-\omega}(p)\right]=1-H(p) .
\end{aligned}
$$

The first equation is again independent of $F$ and coincides what is found in relativistic theories, namely, when propagated backwards in time, pairs of characteristics pill up exponentially in PG time. The second equation tells us that the "center of mass" of a pair which is centered on the relativistic horizon for $1-H \ll 1$, i.e. $p \ll \Lambda$, gradually moves away as $p$ increases. For sub (super) luminal dispersion, $H<1(H>1)$, the pair is sent outwards (inwards). For quartic dispersion the momentum at the turning point is given by $p_{t . p .}^{3}=2 \Lambda^{2}|\omega|[34]$. Because of this movement away from the horizon, the increase of $p$ and the focusing of $x_{\omega}-x_{-\omega}$ will stop when the pair reaches $|\kappa x| \sim D$, where the gradient $\partial_{x} w$ drops down. A straightforward calculation gives that the focusing stops for $p \sim \Lambda D^{1 / 2}$.

The end of the focusing and the movement away from the horizon are the principal consequences of dispersion. They imply that the early properties of Fig. 1 are inevitably modified, as we shall see below.

\section{Correlations from wave packets}

To show how dispersion affects the correlation pattern encoded in Eq. (38), we need Eq. (46) in $x$-space

$$
\phi_{\omega}^{i n}(x)=\int_{0}^{\infty} \frac{d p}{(2 \pi)^{1 / 2}} e^{i p x} \tilde{\phi}_{\omega}^{i n}(p) .
$$

Far away from the turning point, one can evaluate this integral at the saddle point approximation (since it is reliable 16, 34]), and decompose Eq. (50) in terms of outgoing modes defined for low momenta. Doing so one finds

$$
\phi_{\omega}^{i n}(x)=\alpha_{\omega}\left[\theta(x) \varphi_{\omega}(x)+z_{\omega} \theta(-x)\left(\varphi_{-\omega}(x)\right)^{*}\right],
$$

where $\varphi_{\omega}(x)$ is the $x$-WKB mode of Eq. (37) with unit norm. Explicitely it is given by

$$
\varphi_{\omega}(x)=\sqrt{\frac{\partial p_{\omega}(x)}{\partial \omega}} \frac{\exp \left(i \int_{x^{0}}^{x} d x^{\prime} p_{\omega}\left(x^{\prime}\right)\right)}{\sqrt{4 \pi \Omega\left(p_{\omega}(x)\right)}},
$$

where $p_{\omega}(x)$ is the corresponding low momentum root of Eq. (36). One easily verifies that these out modes are identical to the relativistic ones for $p_{\omega} \ll \Lambda$. Moreover, for $\kappa \ll \Lambda$, up to a phase, one finds $z_{\omega}=e^{-\pi \omega / \kappa}$. Hence Eq. (51) gives the equivalent of Eq. (A2). ${ }^{6}$

In addition to the above two low momentum modes, there is a third saddle -on the left (right) of the horizon for super (sub) luminal dispersion, in conformity with Eq. (49)- which gives a high momentum mode. Its WKB wave is also given by Eq. (52) with $p_{\omega}$ being the unique large positive real root of Eq. (36). One verifies that its overall coefficient is unity in conformity with the fact that it describes the incoming mode that shall be scattered.

Therefore, considering Eq. (38) with $\bar{f}_{\omega}$ centered around $0<\bar{\omega} \ll \omega_{\max }$, we get two results. First, at late times, using Eq. (51) and Eq. (52), one finds two low momentum packets following Eq. (49) with $\omega= \pm \bar{\omega}$, where the negative frequency packet has its amplitude reduced by $z_{\bar{\omega}}$, as in Eq. (A3). Since $z_{\omega}=e^{-\pi \omega / \kappa}$, and since both $\varphi_{\omega}$ of Eq. (51) behave as relativistic modes once $p$ is small enough $(F-p \ll p)$, at large distances, the pattern is indistinguishable from the relativistic one obtained by replacing Eq. (46) by Eq. (45).

Second, at early times, only the incoming high momentum mode constructively interferes. It has a mean positive frequency $\bar{\omega}$, follows the second line of Eq. (49) with $p \gg \bar{\omega}$, and leaves the near horizon region with $p \sim \Lambda$. This is completely different from what is obtained in the relativistic case. Indeed, using Eq. (45), $p$ would keep increasing for ever following Eq. (48), and the spread in $x$ correspondingly decrease as $\sim 1 / p$.

\footnotetext{
6 This approximation is valid provided $\omega$ is sufficiently small. For quartic dispersion there is a critical frequency $\omega_{\max }$, related to both $\Lambda$ and the asymptotic velocities $w( \pm \infty)$, above which $z_{\omega}$ identically vanishes [35]. From the numerical results of that ref., a good fit is $\left|z_{\omega}^{F}\right|=e^{-\pi \omega / \kappa}\left(1-\omega / \omega_{\max }\right)^{1 / 4}$.
} 
From the analysis of wave packets, we have thus reached two important results. On one hand, the low momentum (late time) properties of the relativistic pattern of Fig. 1 is unaffected by dispersion. On the other hand, the early properties of this pattern will be radically affected by dispersion since the peak of correlations will follow Eq. (49), as represented in Fig. 4 of [16]. To further investigate how dispersion affects the properties of Figs. 1 and 2, we now consider the pattern encoded in Eq. (39) rather than in Eq. (38).

\section{Correlations in energy density}

We start with the correlations of a relativistic field expressed in the present language. Since $\Omega=p$, the correlation function of $\rho$ of Eq. (41) is

$$
\left\langle\rho(x, \tau) \rho\left(x_{0}, \tau_{0}\right)\right\rangle_{i n}=\left(\partial_{x} \partial_{x_{0}} \int_{-\infty}^{\infty} d \omega e^{-i \omega\left(\tau-\tau_{0}\right)} G_{\omega}^{i n}\left(x, x_{0}\right)\right)^{2},
$$

where $G_{\omega}^{i n}=\phi_{\omega}^{i n}(x)\left(\phi_{\omega}^{i n}\left(x_{0}\right)\right)^{*}$ is the $\omega$ component of $G^{i n}$ of Eq. (39). In the $p$-representation, using Eq. (45), one gets

$$
\begin{aligned}
\tilde{G}_{K}\left(p, \tau ; p_{0}, 0\right) & =\int_{-\infty}^{\infty} d \omega e^{-i \omega \tau} \tilde{G}_{\omega}^{i n}\left(p, p_{0}\right) \\
& =\theta(p) \theta\left(p_{0}\right) \frac{1}{2 p p_{0}} \delta\left(\kappa \tau+\ln \left(p / p_{0}\right)\right) .
\end{aligned}
$$

On one hand, we recover the classical evolution law of Eq. (48). On the other we learn that in the Unruh-vacuum, at $\tau=\tau_{0}$, only configurations with equal values of $p$ contribute. There is no spread in $p$ in this state. When inverse Fourier transform, one gets

$$
G_{K}\left(x, \tau ; x_{0}, 0\right)=-\frac{1}{4 \pi} \ln \left(x-x_{0} e^{\kappa \tau}+i \epsilon\right) .
$$

We recover the standard result, Eq. (B5), expressed in PG coordinates. At equal PG time, we notice also that the argument of the $\log$ is $x-x_{0}$. We shall return to this point below. When computing $\partial_{x} \partial_{x_{0}} G_{K}$ one obtains

$$
\partial_{x} \partial_{x_{0}} G_{K}\left(x, \tau ; x_{0}, 0\right)=-\frac{1}{4 \pi} \frac{e^{\kappa \tau}}{\left(x-x_{0} e^{\kappa \tau}+i \epsilon\right)^{2}},
$$

which is the square root of Eq. (19) in the near horizon region where $\mathcal{U}_{K} \sim-x$.

When introducing dispersion, $\rho$ receives corrections with respect to $\left(\partial_{x} \phi\right)^{2}$ due to the non-linearities of $F^{2}$. This is hardly relevant for us, because in the near horizon region, the momenta $p$ are much smaller than the UV scale $\Lambda$. They are of course modifications in the UV sector of the theory, but these ultra local effects are the same as in Minkowski space. Therefore the main modifications will come from the replacement of the Unruh modes by the modified ones. Using Eq. (46), Eq. (54) is replaced by ${ }^{7}$

$$
\tilde{G}^{i n}\left(p, \tau ; p_{0}, 0\right)=\tilde{G}_{K}\left(p, \tau ; p_{0}, 0\right) \times \frac{\exp i \int_{p_{0}}^{p}\left[H\left(p^{\prime}\right)-1\right] d p^{\prime} / \kappa}{\left(H(p) H\left(p_{0}\right)\right)^{1 / 2}} .
$$

It should be noticed that the frequency $\omega_{\max }$ mentioned in footnote 6, will cut out the integral in Eq. (53), below $-\omega_{\max }$ for superluminal dispersion, and above $\omega_{\max }$ for sub luminal. We ignored for this UV cutoff in computing Eq. (57) because Eq. (46) is no longer trustworthy anyway when $\omega \rightarrow \omega_{\max }$. In fact, in the adiabatic approximation of Eq. (47), there are cancelling errors, in that the next equation can be shown to be exact.

\section{Equal time correlations}

At equal times, Eq. (57) gives

$$
\tilde{G}^{i n}\left(p, p_{0}, \delta \tau=0\right)=\theta(p) \frac{\delta\left(p-p_{0}\right)}{2 \Omega(p)}
$$

\footnotetext{
7 We proceed as in 15. Nevertheless the forthcoming equations differ in several respects.
} 
and in the $x$-representation, one has

$$
G^{i n}\left(x, x_{0}, \delta \tau=0\right)=\int_{0}^{\infty} \frac{d p}{4 \pi} \frac{e^{i p\left(x-x_{0}\right)}}{\Omega(p)} .
$$

The only effect of dispersion is to replace in the denominator the relativistic law $\Omega=p$ by $\Omega=F(p)$. Therefore Eq. (58) is exactly what one obtains in Minkowski vacuum in the preferred frame. The reason is again that $x$ is affine at fixed $\tau: d s^{2}=d x^{2}$. In fact, as in the relativistic case, see Sec. III.F, a non-stationary vacuum can be defined at a given time but for all values of $x$ by plane waves $e^{i p x}$ with $p>0$. Then, the negligible character of the non-adiabatic corrections to Eq. (47) in the near horizon region gives Eq. (57) which implies that in that region but at all times the vacuum stays characterized by $p>0 .{ }^{8}$ This also implies that the dispersive version of the time-dependent Eq. (30) will evolve towards the stationary Eq. (39), as Eq. (30) evolved into the stationary function in the Unruh vacuum.

From Eq. (59) several consequences can be drawn. If one probes the in state for $\kappa|x| \ll 1$, the deviations w.r.t. to the relativistic case for $x-x_{0}<1 / \Lambda(p>\Lambda)$ are the same as in Minkowski, and are therefore insensitive to presence of the black hole. If one probes the $i n$ state further away from the horizon and for momenta $p<\Lambda$, since $\Omega \sim p$, Eq. (39) will behave as the relativistic function, as it obeys the same equation, and possesses the same initial conditions. Hence the whole analysis of Sec. III.E.2 applies. In particular, as soon as $w$ is constant, $\partial_{x} w \ll \kappa$, Eq. (53) will obey Eq. (27) when both points are on the same side of the horizon, and Eq. (28) when one is on either side. ${ }^{9}$ Thus the properties of Fig. 2 (left) are not affected by dispersion when $\kappa \ll \omega_{\max }$. Those of Fig. 2 (right) are not either when subtracting the dispersive expression that replaces the $\log$ in Eq. (29), because $S_{K}$ varies on scales $1 / \kappa \gg 1 / \Lambda$.

The insensitivity of Fig.2 against introducing dispersion is quite surprising since, as discussed before, we expect that the properties of Fig. 1 be affected by the drift of Eq. (49) which occurs for rather low momenta $\sim \Lambda^{2 / 3} \kappa^{1 / 3} \ll \Lambda$. The reason of the disappearance of the drift (at equal PG time) is the following. In Eq. (53), because we are summing over $\omega$, we erase the coherence in $x$-space that exists in each $\omega$ sector, thereby recovering the translation invariance of the in state, as in Eq. (B5). In other words, it is only when isolating some $\omega$ content out of all vacuum configurations that the early pattern characteristic of wave packets emerges. This deserves further comments.

Given Eq. (59), what can be said about the entanglement entropy? The regular behavior of the dispersive in modes and the entanglement in Fock space between states of opposite $\omega$, see Eq. (B2), were exploited in [30] to argue that the entanglement entropy of a black hole is finite (in 1+1 dimensions). However, using Eq. (59) one would conclude that upon tracing over inside configurations $x<0$, one would obtain the same (diverging) result as in Minkowski [37]. This conflictual result indicates that there is probably no unique notion of the entanglement entropy. Therefore to get a well defined result, it is needed to specify what one exactly means by "tracing over the inside configurations".

We saw that the 2pt correlation function (at equal time) does not display the characteristic pattern of wave packets with a given frequency content. This is quite general. It was discussed in $[6]$ when studying the correlations amongst particles emitted by accelerator mirrors, and in a inflationary context in [26]. ${ }^{10}$

\section{Correlations at different times}

When $\tau \neq 0$, in the relativistic case one gets Eq. (55). Instead, Eq. (57) gives

$$
G^{i n}\left(x, \tau ; x_{0}, 0\right)=\int_{0}^{\infty} \frac{d p}{4 \pi} e^{i p \delta} \frac{e^{\kappa \tau / 2}}{\left[\Omega(p) \Omega\left(p e^{\kappa \tau}\right)\right]^{1 / 2}} \exp i \int_{p e^{\kappa \tau}}^{p}\left[H\left(p^{\prime}\right)-1\right] d p^{\prime} / \kappa,
$$

where $\delta=x-x_{0} e^{\kappa \tau}$. The non-trivial modifications of the correlations due to dispersion are best seen by evaluating this integral at the saddle point approximation. The value of the saddle $p_{*}$ answers the classical question: given that one starts at $x_{0}, \tau_{0}=0$ and ends at $x, \tau$, what is the momentum at that time ? It is given by

$$
\kappa \delta=\left[e^{\kappa \tau}\left(H\left(p_{*} e^{\kappa \tau}\right)-1\right)-\left(H\left(p_{*}\right)-1\right)\right] .
$$

8 Eq. (58) is also obtained in the adiabatic approximation, in cosmological backgrounds when the preferred frame is aligned along the cosmic frame. Moreover, this correspondence becomes exact (beyond the adiabatic approximation) when considering de Sitter space when the Hubble parameter $H=\kappa$ since the linearized expression $w=-1+\kappa x$ describes this space in PG coordinates when $-\infty<x<\infty$.

${ }^{9}$ In this we recover what has been found in Bose Einstein condensates when looking at the density-density correlation function [18, 19]. In that case, in the hydrodynamical limit, the atom density fluctuation is given by $\partial_{x} \phi$, and the correlation corresponds to Eq. (56).

10 These remarks raise the question of the choice of (the set of) observables used to probe a quantum state. To give a concrete ex.: in inflationary cosmology, it is generally assumed that the large amplification experienced by primordial fluctuations erase all quantum properties and would give a state indistinguishable from a stochastic ensemble of classical fluctuations. In [38] it was shown that irrespectively of the amplification there exist observables exhibiting violations of Bell inequalities (for linearized modes). 
To understand the implications of this expression, we consider three regimes. First, if $\kappa \delta \ll 1$ and $\kappa \tau \sim 1$, the two points are almost connected by a null ray and the red-shifting effect is moderate. In this case $p_{*} / \Lambda \ll 1$ and one recovers the relativistic behavior of Eq. (55).

Second, we study the non-trivial correlation far away from the light cone (but still in the near horizon region). For definiteness we restrict attention to quartic laws $F^{2}=p^{2} \pm p^{4} / \Lambda^{2}$. We expand Eq. (61) to first order in $1 / \Lambda^{2}$, and using $H-1 \sim \pm p^{2} / 2 \Lambda^{2}$, we get

$$
\kappa \delta= \pm \frac{p_{*}^{2}}{2 \Lambda^{2}}\left(e^{3 \kappa \tau}-1\right)
$$

When $x=x_{0}>0$, irrespectively of the sign of $\tau$, there is no (real) saddle for the + sign, i.e., superluminal dispersion, in agreement with Eq. (49) which says that both partners are dragged inside the black hole horizon. Instead, for subluminal dispersion, since they are both dragged outside, there must be a non trivial solution. To confirm this, we take $\kappa \tau$ such that $e^{\kappa \tau} \gg e^{-\kappa \tau}$. In this regime, Eq. (62) reduces to $\kappa x_{0}=p_{*}^{2} e^{2 \kappa \tau} / 2 \Lambda^{2}$. From this we can deduce $\omega_{*}$ the mean value of the frequency corresponding to the trajectory that goes from $x_{0}$ back to it in a lapse equal to $\tau$. It is approximatively given by

$$
\omega_{*}=\sqrt{2} \Lambda\left(\kappa x_{0}\right)^{3 / 2} e^{-\kappa \tau} .
$$

This result can also be derived using Eq. (49) (and applied to superluminal dispersion for $x<0$ ). Thus, when studying $G\left(x, \tau ; x_{0}, 0\right)$ at sufficiently large $\kappa \tau$, unlike what we found in Eq. (59), the correlations are now in agreement with the locus of constructive interferences of wave packets because only a limited range of frequencies centered about $\omega_{*}$ significantly contributes. This confirms that near horizon behavior of Eq. (53) will completely differ from that of Fig. 1, and will be similar to those of Fig. 1. of [30]. What remains to be clarified concerns the profile of Eq. (53) at early times. Namely, at fixed $x_{0}, \tau_{0}$, what is the trajectory of the maximum of Eq. (53), and what is its spread in $x$ as a function of $x_{0}, \tau-\tau_{0}$, and $\Lambda$ ? We conjecture that both of these quantities are ruled by $\omega_{\max }$ of footnote 6 .

What can be studied [15] is the "off-shell" limit of very large blue-shift $e^{\kappa \tau} \gg 1$ encoded in a backward propagation at fixed $x$ and fixed $\delta=x-x_{0} e^{\kappa \tau}$. This limit displays how dispersion tames the "trans-Planckian" behavior found for the relativistic field. In that case, Eq. (56) gives

$$
\partial_{x} \partial_{x_{0}} G_{K}\left(x, 0 ; x_{0},-\tau\right)=-\frac{1}{4 \pi} \frac{e^{\kappa \tau}}{(\delta+i \epsilon)^{2}},
$$

how ever large is $\kappa \tau>0$, in agreement with Fig. 1. In the dispersive case, using Eq. (60) and $H(p)-1 \sim \pm p^{2} / 2 \Lambda^{2}$, one has

$$
\partial_{x} \partial_{x_{0}} G^{i n}\left(x, 0 ; x_{0},-\tau\right)=\int_{0}^{\infty} \frac{d p}{4 \pi} \frac{p^{2} e^{3 \kappa \tau / 2}}{\left[\Omega(p) \Omega\left(p e^{\kappa \tau}\right)\right]^{1 / 2}} \exp i\left(p \delta \mp \frac{p^{3} e^{3 \kappa \tau}}{6 \Lambda^{2} \kappa}\right) .
$$

When the blue shift is moderate, i.e. $e^{\kappa \tau}<\kappa \delta(\Lambda / \kappa)^{2 / 3}$, Eq. (65) behaves as Eq. (64) plus corrections in $e^{3 \kappa \tau} / \Lambda^{2} \kappa \delta^{3} \ll$ 1 that can be computed perturbatively, as can be seen by changing variable $p \rightarrow q=p \delta$. Instead, when the blue shift is large: $e^{\kappa \tau}>\kappa \delta(\Lambda / \kappa)^{2 / 3}$, the integral becomes independent of $\delta$ as is seen by using $k=p e^{\kappa \tau} /\left(\Lambda^{2} \kappa\right)^{1 / 3}$. Explicitly one finds

$$
\partial_{x} \partial_{x_{0}} G^{i n}\left(x, 0 ; x_{0},-\tau\right) \sim\left(\Lambda^{2} \kappa\right)^{2 / 3} e^{-\kappa \tau} \times C_{ \pm}(\kappa / \Lambda, \tau),
$$

where $C_{ \pm}(\kappa / \Lambda, \tau)$ are slowly varying functions which stay bounded for $\tau \rightarrow \infty$. This exponentially decreasing result can be seen as the contribution on the horizon of the tail of the configurations with high $p$ which follow the second equation in Eq. (49).

This smoothing out of the relativistic behavior is very reminiscent to what was found in [21] when studying the backwards evolution of $G^{i n}\left(x, 0 ; x_{0},-\tau\right)$ of a relativistic field propagating in a stochastically fluctuating black hole metric. In addition, for nearby points, $G^{i n}$ in a stochastic geometry also behaved as Eq. (59), as can be seen in Eq. (4.7). Based on this similarity it was argued [22] that when taking into account the gravitational radiative corrections, the dressed Green functions should effectively behave near a black hole horizon as in Eq. (66), thereby reinforcing the idea that the unbounded growth of Eq. (64) cannot "accommodate gravitational non-linearities".

\section{CONCLUSIONS}

We showed that the monotonic energy correlations found in the vacuum Eq. (1) gives rise to a maximum of correlation across a Rindler horizon when re-expressed in terms of coordinates associated with accelerated systems, 
see Eq. (3). This maximum is not a mere coordinate artefact as it affects the combined state of co-accelerating systems.

In Sec. III, we transposed this analysis to stationary black hole geometries, and recalled that the regularity of the state across the horizon and the inertial character of asymptotic observers are essential to provide a physical meaning to the thermal correlations of Eq. (2). When considering black hole geometries which contain asymptotic regions on both sides of the horizons, the correlations of Eq. (3) are found at large distances when using inertial coordinates. We then make use of the affine parameter $r_{v}$ of Eq. (11) to obtain an invariant description of the energy correlations in the entire space-time. We compared the correlation pattern associated with a late detection, Fig. 1, to that obtained at equal EF time, Fig. 2. In both cases the gradual emergence of a maximum of correlations across the horizon is clearly visible. By considering the subtracted correlations of Eq. (29), we saw that the remaining signal is dominated by the long distance correlations across the horizon, and also contains a sub-dominant local contribution associated with Eq. (2). This analysis was generalized in III.F. by including the transients effects which precede the stationary patterns found in the Unruh vacuum.

In Sec. IV. by studying both wave packets and correlation functions, we studied how these patterns are modified by dispersion. Far away from the horizon, the pattern is robust, i.e. hardly affected by dispersion. Close to the horizon we saw that dispersive effects show up differently depending on how one probes the state. When probed at equal PG time, the correlation function is translation invariant, and as in Minkowski, see Eq. (59). Instead wave packets of $i n$ modes centered around a given frequency $\omega$ display a characteristic pattern which follows the modified characteristics of Eq. (49). When the momentum has sufficiently increased (in a backward in time propagation) the wave packets are dragged away from the horizon, and, the blue shift effect saturates. This behavior is recovered from the correlation function when considered at different times and appears through a non-trivial saddle point in Eq. (60). When considering the correlation function for two points separated by a very large PG time, the drag w.r.t. the relativistic horizon results in an exponentially suppressed amplitude in the place of the exponentially growing result found in relativistic theories, compare Eq. (64) with Eq. (65). These properties are reminiscent to what was found when considering field propagation in a fluctuating black hole metric, and could possibly be found when taking into account gravitational interactions at the quantum level.

\section{Acknowledgments}

We would like to thank T. Jacobson for discussions concerning the affinity of $r$, and R. Balbinot, S. Finazzi and N. Obadia for useful remarks.

\section{Appendix A: Unruh modes}

Firstly, they are solutions of d'Alembert equation $\partial_{U} \partial_{V} \phi_{\omega}=0$, and thus only depend on either $U$ or $V$. Secondly, they have a fixed boost frequency $\omega$, i.e. they are eigenmodes of

$$
i \partial_{u} \phi_{\omega}=-i a U \partial_{U} \phi_{\omega}=\omega \phi_{\omega} .
$$

Thirdly they are only composed of the positive norm modes: $\phi_{\Omega}=e^{-i \Omega U} /(4 \pi \Omega)^{1 / 2}$ with $\Omega>0$. Explicity they are given by

$$
\begin{aligned}
\phi_{\omega} & =\frac{\alpha_{\omega}}{(4 \pi \omega)^{1 / 2}}(-a U+i \epsilon)^{i \omega / a} \\
& =\frac{\alpha_{\omega}}{(4 \pi \omega)^{1 / 2}}\left[\theta(-U)(-a U)^{i \omega / a}+z_{\omega} \times \theta(U)(a U)^{i \omega / a}\right],
\end{aligned}
$$

where the normalization obeys $\left|\alpha_{\omega}\right|^{2}=\left(1-e^{-2 \pi \omega / a}\right)^{-1}$, and where $z_{\omega}=e^{-\pi \omega / a}$. This factor arises from the $i \epsilon$ prescription which specifies that the analytic continuation from the $R$ to $L$ quadrant must be done in lower half complex $U$ plane. As in Eq. (10) this prescription comes from the fact that only positive frequency $\Omega=i \partial_{U}$ modes contribute.

Moreover, they are globally defined, $-\infty<U<\infty$, and form a complete and orthonormal basis of positive norm modes when $-\infty<\omega<\infty$ (with respect to the standard Klein-Gordon product). Hence the Minkowski vacuum can be alternatively defined as the state annihilated by the destruction operators $a_{\omega}$ associated with these modes.

Thus, when a quantum system is (linearly) coupled to $\phi$ which is initially in the vacuum, the transition amplitudes will contain some (linear) combination of the $\phi_{\omega}$. When the system is not accelerated (e.g. inertial), the decomposition (A2) presents no interest since the system will cross $U=0$. On the contrary, when it is uniformly accelerated in, say, 
the $R$ quadrant, Eq. (A2) guarantees that every transition occurring in the Minkowski vacuum defines a partner wave in $L$, see App. C. for more details.

This $R-L$ partnership can be studied in simpler terms and without referring to accelerated systems by constructing wave packets of Unruh modes

$$
\bar{\phi}=\int_{0}^{\infty} d \omega \bar{f}_{\omega} \phi_{\omega}=\bar{\phi}^{R}+\bar{\phi}^{L} .
$$

Eq. (A2) thus implies that to every packet $\bar{\phi}^{R}$ localized in $R$ will correspond $\bar{\phi}^{L}$, its partner wave in $L$. More can be said: since $z_{\omega}$ in the r.h.s. is real for all $\omega$, when $\bar{\phi}^{R}$ constructively interferes around some $\bar{U}_{R}<0$, Eq. (A2) guarantees that $\bar{\phi}^{L}$ will do so near $-\bar{U}_{R}$. This explains why the maximum in Eq. (3) arises for opposite values of $U$. In addition, from the fact that high $\omega$ are exponentially suppressed by $z_{\omega}=e^{-\pi \omega / a}$, the maximum in Eq. (3) cannot diverge as it does in Eq. (2). Instead it must scale as $a^{4}$. In brief, the mathematical properties of Eq. (3) are deeply rooted to those of the modes $\phi_{\omega}$.

There exists an efficient way to encode the properties of the Unruh modes which turns out to be very useful when analyzing Hawking radiation in the presence of dispersion. It consists in computing the Fourier transform at fixed $t$ : $\tilde{\phi}_{\omega}(p)=\int d z e^{-i p z} \phi_{\omega} /(2 \pi)^{1 / 2}$. Taking into account the $i \epsilon$ in Eq. (A2), up to an irrelevant phase, one finds

$$
\tilde{\phi}_{\omega}(p)=\theta(p) \frac{p^{-i \omega / a-1}}{(4 \pi a)^{1 / 2}} .
$$

The restriction to positive $p$ follows from the fact that property only positive $\Omega$ contribute to $\phi_{\omega}$, and from the dispersion relation $\Omega=p$ which describes right moving modes. We also note that when considering Eq. (A3) in the $p$-representation, the two wave packets found in the $x$-representation on either side the horizon are now described by a single packet in $p$-space. This is characteristic of pair production phenomena, see e.g. Sec. 1.2-1.3 in [5]. We shall return to this in Sec. IV.

\section{Appendix B: Fulling-Rindler states and partner-ship in Fock space}

If a mode analysis is sufficient to understand the behavior of Eq. (3) in space-time, to have a deeper quantum mechanical understanding of Eq. (3) we analyze the bi-partite structure in the Fock space when using states with a fixed frequency $\omega$. To this end, we should discuss yet another property of Eq. A2). It concerns the fact that the mode on the left $(U>0)$ has a negative norm (for $\omega>0)$ thereby implying that the norm of the right component is correspondingly larger. This invites to consider the inequivalent quantization of $\phi$ based on the "Fulling-Rindler" (FR) modes. These are normalized eigenmodes of frequency $\omega$, Eq. (A1), localyzed either on the right, or the left, of $U=0$. Hence we re-write Eq. (A2) as

$$
\begin{aligned}
& \phi_{\omega}=\alpha_{\omega} \phi_{\omega}^{R}+\beta_{\omega}\left(\phi_{\omega}^{L}\right)^{*}, \quad \text { for } \omega>0, \\
& \phi_{\omega}=\alpha_{-\omega} \phi_{-\omega}^{L}+\beta_{-\omega}\left(\phi_{-\omega}^{R}\right)^{*}, \quad \text { for } \omega<0,
\end{aligned}
$$

where $\phi_{\omega}^{R}=e^{-i \omega u} /(4 \pi \omega)^{1 / 2}\left(\phi_{\omega}^{L}=e^{-i \omega \bar{u}} /(4 \pi \omega)^{1 / 2}\right)$ vanishes on the left (right) of the horizon, and where $\beta_{\omega}=z_{\omega} \alpha_{\omega}$. One easily verifies that $\alpha_{\omega}^{2}-\beta_{\omega}^{2}=1$, which implies $\beta_{\omega}^{2}=\left(e^{2 \pi \omega / a}-1\right)^{-1}$. For each $\omega>0$, Eq. (B1) defines a (two-mode) Bogoliubov transformation relating $\left(\phi_{\omega}, \phi_{-\omega}\right)$ to $\left(\phi_{\omega}^{R}, \phi_{\omega}^{L}\right)$. This implies that the vacuum can be written as a product over $\omega>0$ of two-mode squeezed states

$$
|0\rangle=\Pi_{\omega}\left(\frac{1}{\alpha_{\omega}} \exp \left(z_{\omega} a_{\omega}^{R} a_{\omega}^{L}\right)^{\dagger}\right)|0\rangle_{R}|0\rangle_{L}
$$

where the $R$-vacuum $|0\rangle_{R}$ is the state annihilated by the $a_{\omega}^{R}$, the destruction operators associated with the $\phi_{\omega}^{R}$, and similarly for the $L$ sector. Since the squeezing operator is quadratic and diagonal in $\omega$, for free fields, all expectation values are expressible in terms of the following two VEV

$$
\begin{aligned}
\left\langle\left(a_{\omega}^{R}\right)^{\dagger} a_{\omega}^{R}\right\rangle & =\left\langle\left(a_{\omega}^{L}\right)^{\dagger} a_{\omega}^{L}\right\rangle=\left|\beta_{\omega}\right|^{2}, \\
\left\langle a_{\omega}^{R} a_{\omega}^{L}\right\rangle & =\beta_{\omega} \alpha_{\omega}^{*}=z_{\omega}\left|\alpha_{\omega}\right|^{2} .
\end{aligned}
$$

It is now instructive to see how these two VEV enter in Eq. (2) and Eq. (3). To this end, we consider the $(U$ contribution of the) 2 point function of $\phi$

$$
\left\langle\phi(U) \phi\left(U_{0}\right)\right\rangle=\int_{-\infty}^{\infty} d \omega \phi_{\omega}(U)\left(\phi_{\omega}\left(U_{0}\right)\right)^{*}=-\frac{1}{4 \pi} \ln \left(U-U_{0}-i \epsilon\right) .
$$


Using Eq. (B1) two different expressions are obtained depending if both points are on one side, or on either side, of $U=0$. Explicitely, when both $U$ are negative and written as $-a U=e^{-a u}$ one has

$$
\left\langle\phi(U) \phi\left(U_{0}\right)\right\rangle=\int_{0}^{\infty} \frac{d \omega}{4 \pi \omega}\left(\left|\alpha_{\omega}\right|^{2} e^{-i \omega\left(u-u_{0}\right)}+\left|\beta_{\omega}\right|^{2} e^{+i \omega\left(u-u_{0}\right)}\right) .
$$

Instead when one point, say $U$, is positive and written as $a U=e^{a \bar{u}}$, one has

$$
\left\langle\phi(U) \phi\left(U_{0}\right)\right\rangle=\int_{0}^{\infty} \frac{d \omega}{4 \pi \omega} 2 \operatorname{Re}\left(\left|\alpha_{\omega}\right|^{2} z_{\omega}^{*} e^{-i \omega\left(\bar{u}+u_{0}\right)}\right) .
$$

One sees that Eq. (B6) and Eq. (21) arise from diagonal terms, hence weighted by $\left|\beta_{\omega}\right|^{2}$ of Eq. (B3), whereas Eq. (B7) and Eq. (3) arise from interfering terms weighted by $\alpha_{\omega}^{*} \beta_{\omega}$ of Eq. (B4) which encodes the entanglement, in Fock space, amongst the $R$ and $L$ sectors.

It is an interesting exercice to verify that when using the actual functions for $\alpha_{\omega}$ and $\beta_{\omega}$, Eq. (B6) and Eq. (B7) both give back, as they must, the $\log \Delta U$ of Eq. (B5). Therefore, they are only complicated re-expressions of Eq. (B5). Similarly, Eq. (B2) is only a mathematical re-expression of the Minkowski vacuum. However, it prepares the analysis of the physical processes related to the Unruh effect, to the quantum fluxes emitted by non-uniform mirrors [3, 6], and to black hole physics. In these three cases, there is an external agent -respectively an accelerated system, a non-uniform mirror, a non-trivial metric- which acts on the field and "transforms" the FR states into asymptotic states. This particularly neat in the case of the non-uniform mirror discussed in [3] , see Eqs. (3.27-3.28), see also Section 2.5 in [5].

\section{Appendix C: The conditional value associated with a detection in $R$}

We recall how a detection of a FR quantum in $R$ defines first, a partner state in $L$, and second, a projector which allows to define the conditional value of an operator associated with this detection. We describe the detected quantum in $R$ by

$$
\left|\bar{\Psi}_{R}\right\rangle=\int_{0}^{\infty} d \omega f_{\omega}\left(a_{\omega}^{R}\right)^{\dagger}|0\rangle_{R}
$$

The EPR partner state is defined by reducing the bi-partite state. In the present case, the latter is the Minkowski vacuum expressed as Eq. (B2). The partner state is thus

$$
\left|\bar{\Psi}_{L}\right\rangle=\left\langle\bar{\Psi}_{R} \mid 0\right\rangle=\int_{0}^{\infty} d \omega z_{\omega}^{*} f_{\omega}^{*}\left(a_{\omega}^{L}\right)^{\dagger}|0\rangle_{L}
$$

One notices that its Fourier components are fixed by $z_{\omega}^{*}$ and $f_{\omega}^{*}$, i.e. by both the state and the complex conjugated of the component of selected wave packet.

It is instructive to relate these two states to the (classical) wave packets of Eq. (A3). To this end we introduce the projector $\Pi_{R}=\left|\bar{\Psi}_{R}\right\rangle\left\langle\bar{\Psi}_{R}\right|$ and consider the value of $\phi^{2}$ conditional to the fact that the detection took place (for more details see [5])

$$
\bar{\phi}^{2}=\left\langle 0\left|\phi^{2} \Pi_{R}\right| 0\right\rangle=\left(\bar{\phi}^{2}\right)^{R}+\left(\bar{\phi}^{2}\right)^{L} .
$$

A direct calculation gives

$$
\begin{aligned}
\left(\bar{\phi}^{2}\right)^{R} & =\left(\int_{0}^{\infty} d \omega f_{\omega} \phi_{\omega}^{R}\right)\left(\int_{0}^{\infty} d \omega f_{\omega}\left|z_{\omega}\right|^{2} \phi_{\omega}^{R}\right)^{*}, \\
\left(\bar{\phi}^{2}\right)^{L} & =\left|\int_{0}^{\infty} d \omega f_{\omega}^{*} z_{\omega}^{*} \phi_{\omega}^{L}\right|^{2} .
\end{aligned}
$$

When $f_{\omega}=\alpha_{\omega} \bar{f}_{\omega}$ where $\bar{f}_{\omega}$ given in Eq. (A3), $\left(\bar{\phi}^{2}\right)^{L}$ exactly gives $\left|\bar{\phi}_{L}\right|^{2}$ of that equation. Similarly the first factor in the first line is $\bar{\phi}_{R}$. The second factor is not its complex conjugated due to the presence of $\left|z_{\omega}\right|^{2}$ in the integrand. However for wave packets with a small spread $\omega$ wrt $a$, this quantum mechanical feature (whose consequences are discussed in [10]) does not significantly affect the spatial properties of the $R$ wave packet. Thus we basically recover the modulus square of the two packets of Eq. (A3). 
The lesson of this exercise is that the pattern obtained by constructing wave packets of Unruh modes as in Eq. (A3) offers a reliable description of the quantum correlations across a Rindler horizon (when the spread in $\omega$ is small enough). This transposes in black hole metrics (without and with dispersion) and implies that the correlation patterns of $i n$ modes also offer a reliable description of the quantum correlations across the horizon.

Finally, we mention that the study of highly excited coherent states, see App. C of [20], offers another way to relate the packets of Eq. (A3) to quantum states. Using these coherent states, one can verify the agreement of both descriptions in describing the $R-L$ correlations.

[1] W. G. Unruh, Phys. Rev. D 14, 870 (1976).

[2] W. G. Unruh and R. M. Wald, Phys. Rev. D 29, 1047 (1984).

[3] R. D. Carlitz and R. S. Willey, Phys. Rev. D 36, 2327 (1987), and 2336 (1987).

[4] S. Massar and R. Parentani, Phys. Rev. D 54, 7426 (1996).

[5] R. Brout, S. Massar, R. Parentani and Ph. Spindel, Phys. Rept. 260, 329 (1995).

[6] N. Obadia and R. Parentani, Phys. Rev. D 67, 024022 (2003), and 024021 (2003).

[7] S. Massar and P. Spindel, Phys. Rev. D 74, 085031 (2006).

[8] S. W. Hawking, Commun. Math. Phys. 43, 199 (1975) [Erratum-ibid. 46, 206 (1976)].

[9] P. C. W. Davies, S. A. Fulling and W. G. Unruh, Phys. Rev. D 13, 2720 (1976).

[10] S. Massar and R. Parentani, Phys. Rev. D 54, 7444 (1996), F. Englert, S. Massar and R. Parentani, Class. Quant. Grav. 11, 2919 (1994).

[11] W. G. Unruh, Phys. Rev. D 51, 2827 (1995).

[12] W. G. Unruh, Phys. Rev. Lett. 46, 1351 (1981).

[13] C. Barcelo, S. Liberati, and M. Visser, Living Rev. Relativity 8, 12 (2005).

[14] T. Jacobson, Phys. Rev. D 53, 7082 (1996).

[15] R. Schutzhold and W. G. Unruh, arXiv:1002.1844 [gr-qc].

[16] R. Brout, S. Massar, R. Parentani and P. Spindel, Phys. Rev. D 52, 4559 (1995).

[17] S. Corley and T. Jacobson, Phys. Rev. D 54, 1568 (1996).

[18] R. Balbinot, A. Fabbri, S. Fagnocchi, A. Recati and I. Carusotto, Phys. Rev. A 78, 021603 (2008).

[19] I. Carusotto, S. Fagnocchi, A. Recati, R. Balbinot and A. Fabbri, New J. Phys. 10, 103001 (2008).

[20] J. Macher and R. Parentani, Phys. Rev. A 80, 043601 (2009).

[21] C. Barrabes, V. P. Frolov and R. Parentani, Phys. Rev. D 62, 044020 (2000).

[22] R. Parentani, Int. J. Theor. Phys. 41, 2175 (2002) arXiv:0704.2563, and Phys. Rev. D 63, 041503 (2001).

[23] T. Jacobson, Phys. Rev. Lett. 75, 1260 (1995).

[24] T. Damour and R. Ruffini, Phys. Rev. D 14, 332 (1976).

[25] W. G. Unruh, Phys. Rev. D 15, 365 (1977).

[26] D. Campo and R. Parentani, Phys. Rev. D 70, 105020 (2004).

[27] R. Parentani and T. Piran, Phys. Rev. Lett. 73, 2805 (1994).

[28] T. Jacobson, Phys. Rev. D 44, 1731 (1991), D 48, 728 (1993).

[29] R. Parentani, Class. Quant. Grav. 10, 1409 (1993).

[30] T. Jacobson and R. Parentani, Phys. Rev. D 76, 024006 (2007).

[31] T. Jacobson, Prog. Theor. Phys. Suppl. 136, 1 (1999) arXiv:hep-th/0001085.

[32] T. Jacobson and R. Parentani, Class. Quant. Grav. 25, 195009 (2008).

[33] R. Balbinot, A. Fabbri, S. Fagnocchi and R. Parentani, Riv. Nuovo Cim. 28, 1 (2005) arXiv:gr-qc/0601079.

[34] S. Corley, Phys. Rev. D 57, 6280 (1998).

[35] J. Macher and R. Parentani, Phys. Rev. D 79, 124008 (2009).

[36] R. Balbinot, A. Fabbri, S. Farese and R. Parentani, Phys. Rev. D 76, 124010 (2007).

[37] L. Bombelli, R. K. Koul, J. H. Lee and R. D. Sorkin, Phys. Rev. D 34, 373 (1986).

[38] D. Campo and R. Parentani, Phys. Rev. D 74, 025001 (2006). 